\title{
Manganese transporter Slc30a10 controls physiological manganese excretion and toxicity
}

\author{
Courtney J. Mercadante, ${ }^{1}$ Milankumar Prajapati, ${ }^{1}$ Heather L. Conboy, ${ }^{1}$ Miriam E. Dash, ${ }^{1}$ Carolina Herrera, ${ }^{1}$ Michael A. Pettiglio, \\ Layra Cintron-Rivera, ${ }^{1}$ Madeleine A. Salesky, ${ }^{1}$ Deepa B. Rao, ${ }^{2}$ and Thomas B. Bartnikas ${ }^{1}$
}

'Department of Pathology and Laboratory Medicine, Brown University, Providence, Rhode Island, USA. ²Center for Drug Evaluation and Research, US Food and Drug Administration, Silver Spring, Maryland, USA.

\begin{abstract}
Manganese (Mn), an essential metal and nutrient, is toxic in excess. Toxicity classically results from inhalational exposures in individuals who work in industrial settings. The first known disease of inherited Mn excess, identified in 2012, is caused by mutations in the metal exporter SLC30A10 and is characterized by Mn excess, dystonia, cirrhosis, and polycythemia. To investigate the role of SLC30A10 in Mn homeostasis, we first generated whole-body SIc30a10-deficient mice, which developed severe Mn excess and impaired systemic and biliary Mn excretion. Slc30a10 localized to canalicular membranes of hepatocytes, but mice with liver SIc30a10 deficiency developed minimal Mn excess despite impaired biliary Mn excretion. SIc30a10 also localized to the apical membrane of enterocytes, but mice with SIc30a10 deficiency in small intestines developed minimal Mn excess despite impaired Mn export into the lumen of the small intestines. Finally, mice with SIc30a10 deficiency in liver and small intestines developed Mn excess that was less severe than that observed in mice with whole-body SIc30a10 deficiency, suggesting that additional sites of SIc30a10 expression contribute to Mn homeostasis. Overall, these results indicated that SIc30a10 is essential for $\mathrm{Mn}$ excretion by hepatocytes and enterocytes and could be an effective target for pharmacological intervention to treat Mn toxicity.
\end{abstract}

\section{Introduction}

Acquired from the diet, manganese $(\mathrm{Mn})$ is an essential nutrient. Mn excess can lead to neurological deficits and a disease known as manganism, with psychological and motor disturbances similar but not identical to those seen in idiopathic Parkinson's disease (1, 2). Populations at risk for Mn toxicity include patients with iron deficiency or liver cirrhosis, recipients of Mn-supplemented total parenteral nutrition, and miners and welders who inhale Mn-rich fumes and particulates (2-5). In these populations, regulation of $\mathrm{Mn}$ absorption from the gut is either aberrant or bypassed, or excretion of Mn by the hepatobiliary tract is impaired.

Mn levels are regulated by gastrointestinal (GI) absorption and hepatobiliary excretion. Dietary Mn may be absorbed into duodenal enterocytes by SLC11A2 (also known as divalent metal transporter 1 [DMT1]), however, in vivo data suggest that SLC11A2 is not necessary for Mn absorption (6, 7). Mn may be exported from enterocytes into blood by the iron transporter SLC40A1 (ferroportin), however, evidence for the role of SLC40A1 in Mn homeostasis remains controversial (8-14). The metal importer SLC39A14 (also known as ZIP14) and exporter SLC30A10 (also known as ZNT10) have been implicated in Mn transport across the intestines. In cell culture, SLC39A14 mediates basolateral-to-apical/secretory Mn

Related Commentary: p. 5082

Conflict of interest: The authors have declared that no conflict of interest exists. Copyright: (c) 2019, American Society for Clinical Investigation.

Submitted: April 19, 2019; Accepted: September 10, 2019; Published: November 4, 2019 Reference information: / Clin Invest. 2019;129(12):5442-5461.

https://doi.org/10.1172/JCl129710. transport, and deficiency in SLC39A14 enhances Mn transport in the apical-to-basolateral/absorptive direction (15). Studies in mice with endoderm-specific Slc30a10 deficiency and mice with intestine-specific Slc39a14 deficiency suggest that intestinal Slc30a10 and Slc39a14 regulate systemic Mn levels $(15,16)$. Once absorbed, Mn may be imported into cells by SLC11A2 and other transporters including SLC39A8 (also known as ZIP8), SLC39A14, and the transferrin receptor (17). SLC39A14 is required for Mn import into the liver from blood; SLC39A8 reclaims Mn from bile into the liver (18-21). Deficiency in SLC39A14 results in systemic Mn excess, whereas deficiency in SLC39A8 results in systemic Mn deficiency. SLC30A10 is also expressed in the liver and probably contributes to Mn export from the liver into bile. Although biliary Mn excretion is hypothesized to be the main route for Mn excretion, the molecular mechanisms for Mn excretion in vivo have not yet been fully established.

The first reported inherited disease of Mn excess, SLC30A10 deficiency, was identified in 2012, offering a key opportunity to understand molecular mechanisms of Mn homeostasis (22-24). SLC30A10 is a member of the cation diffusion facilitator superfamily of metal transporters. It is localized to the cell membrane and implicated in Mn export (25). Patients with homozygous mutations in SLC3OA1O develop cirrhosis, dystonia, polycythemia, hypermanganesemia, and brain Mn excess, without a history of environmental Mn exposure, suggesting that Mn excess is caused by mutations in SLC3OA1O. SNPs in SLC3OA1O are associated with changes in blood and dentine Mn levels and neurological function and may influence Mn homeostasis early in development (26-30), further highlighting the idea that SLC30A10 is a key determinant of body Mn levels. 
Since inherited Mn-related diseases were only recently discovered, our understanding of the role of SLC30A10 in Mn homeostasis in vivo is limited. A recent study from the Mukhopadhyay group demonstrated that Slc30a10-deficient mice develop hypothyroidism secondary to Mn excess but did not explore the mechanism linking Slc30a10 deficiency to Mn excess (31). (Hypothyroidism has not been reported in patients with SLC30A10 mutations.) Mukhopadhyay et al. also reported on endodermspecific Slc30a10-deficient mice characterized by increased Mn levels in liver and other tissues and decreased fecal Mn levels (16). These results suggest that endoderm-derived tissues are important for Mn homeostasis and that Slc30a10 may be required for Mn excretion. However, this work did not directly measure excretion, as changes in fecal Mn levels can reflect changes in absorption and excretion. The authors also generated hepatocyte-specific Slc30a10-deficient mice, which developed a minimal phenotype, although they did not assess whether hepatobiliary Mn excretion was impaired in this model.

In this study, we investigated the role of Slc30a10 in Mn homeostasis by generating and characterizing global and tissuespecific mouse models of Slc30a10 deficiency using genetic, metabolic, dietary, and surgical approaches. First, we demonstrated that our mouse model of global Slc30a10 deficiency recapitulates key human disease phenotypes. We then confirmed that Slc30a10 is essential not only for systemic Mn excretion but specifically for hepatobiliary Mn excretion. Given that our mice with hepatocyte-specific Slc30a10 deficiency also developed a minimal phenotype, we then identified the intestines as a prominent site of Slc30a10 expression using a CRISPR-generated mouse line expressing GFP-tagged Slc30a10 from the endogenous gene. We then demonstrated that mice with hepatocyte and enterocyte Slc30a10 deficiency have moderate, but not severe, Mn excess and that enterocyte Slc30a10 deficiency impairs Mn excretion by the small intestines. Overall, our work is the first to our knowledge to establish physiologic and mechanistic roles of Slc30a10 in vivo and indicates that the regulation of Mn levels by Slc30a10 is more complicated than previously anticipated.

\section{Results}

Global Slc30a10 deficiency in mice recapitulates key disease phenotypes. To establish the role of Slc30a10 in Mn homeostasis, we generated mice with global Slc30a10 deficiency $\left(\mathrm{Slc} 30 \mathrm{a} 1 \mathrm{O}^{\mathrm{KO} / \mathrm{KO}}\right)$ by traditional gene targeting using homologous recombination to delete exon 3 and the coding region of terminal exon 4 of Slc30a10 (Supplemental Figure 1, A and B; supplemental material available online with this article; https://doi.org/10.1172/JCI129710DS1). Given that the mutant mice did not consistently survive past 12 weeks of age (data not shown), the $S l c 30 a 10^{K O / K O}$ mice were analyzed at 8 weeks of age. Slc30a10 deficiency was confirmed in tissues known to express Slc30a1O including liver, brain, and duodenum (Figure 1A). Slc3Oa1O ${ }^{K O / K O}$ mice had hepatosplenomegaly, increased brain weights, and decreased body weights (Figure 1, B and C). Hepatosplenomegaly has been reported in patients $(22,24)$. Mn levels were increased in $\mathrm{Slc30} 1 \mathrm{OO}^{\mathrm{KO} / \mathrm{KO}}$ tissues but most notably in liver, bone, brain, and duodenum (Figure 1D and Supplemental Figure 2A). MRI identified T1-weighted hyperintensities in the brain, spinal cord, and abdominal regions of the mutant mice (Figure 1E), which may indicate $\mathrm{Mn}$ accumulation, since $\mathrm{Mn}$ is paramagnetic. We also noted changes in iron, copper, and zinc levels in several tissues, most notably liver and blood (Supplemental Figures 2 and 3). We found that blood Mn levels were increased by roughly 100 -fold in $\mathrm{Slc3Oa1O^{KO } / \mathrm { KO }}$ mice and by 2 -fold in $\mathrm{Slc} 3 \mathrm{Oa} 1 \mathrm{O}^{+/ \mathrm{KO}}$ mice, although the latter increase was not significant (Figure 2A). RBC counts were increased in $\mathrm{Slc} 30 \mathrm{O} 1 \mathrm{O}^{\mathrm{KO} / \mathrm{KO}}$ mice, indicative of polycythemia, as seen in patients (Figure 2B). Several other red cell parameters were greater in the mutant mice (Figure 2, C-F). Thyroxine levels

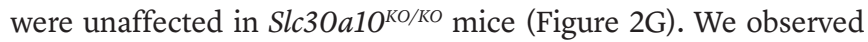
that the thyroid follicle area was decreased in female $\mathrm{Slc3Oa1O^{KO/KO }}$ mice (Figure 2, H and I). Histology of Slc30 $10^{K O / K O}$ liver, duodenum, pancreas, and spleen showed no prominent pathology (Supplemental Figure 4). Overall, recapitulation of phenotypes of human SLC30A10 deficiency - Mn excess, polycythemia, hepatosplenomegaly, and MRI T1 hyperintensities - suggested that Slc3Oa1O $\mathrm{KO} / \mathrm{KO}$ mice are a valid model for interrogation of the function of Slc30a10 in Mn homeostasis.

Hepatobiliary Mn excretion is increased in WT mice loaded with dietary Mn. To understand the role of Slc30a10 in Mn homeostasis, we first evaluated systemic Mn excretion in WT mice on a control $\mathrm{Mn}(10 \mathrm{ppm})$ or high-Mn (2400 ppm) diet from weaning until tissue harvesting at 8 weeks of age. We selected the high-Mn diet on the basis of studies reporting increased tissue Mn levels without apparent toxicity $(18,32)$. These experiments provided a reference for $\mathrm{Mn}$ excretion in a mouse with functional Slc30a10 and Mn excess.

WT mice on a high-Mn diet accumulated $\mathrm{Mn}$ in blood, liver, bone, and other tissues (Figure 3, A and B). However, Mn levels

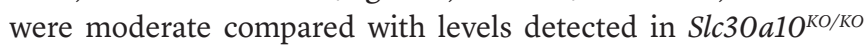
mice (Figure 1D and Figure 2A). The high-Mn diets led to decreased liver Slc30a10 RNA levels in female mice, decreased liver Slc39a8 and Slc39a14 RNA levels in all mice, but no changes in Slc30a10, Slc39a8, or Slc39a14 RNA levels in brain or duodenum (Supplemental Figure 5). Slc30 $10^{\mathrm{KO} / \mathrm{KO}}$ mice also had decreased liver Slc39a14 RNA levels and increased brain Slc39a8 RNA levels (Supplemental Figure 5). The significance of these expression changes is not clear at this time. Although copper and zinc levels were largely unaffected, blood and tissue iron levels were decreased in mice raised on a high-Mn diet, which most likely reflects shared mechanisms for Mn and iron absorption (Figure 3, C-H).

To evaluate systemic Mn excretion in WT mice raised on a high-Mn diet, mice were retro-orbitally injected with ${ }^{54} \mathrm{Mn}$ and housed overnight in metabolic cages for collection of feces and urine. We found that fecal ${ }^{54} \mathrm{Mn}$ levels were elevated in WT mice on a high-Mn diet (Figure $4 \mathrm{~A}$ ). Urinary ${ }^{54} \mathrm{Mn}$ levels were minimal compared with fecal ${ }^{54} \mathrm{Mn}$ levels for all mice analyzed and were unaffected by diet (Figure 4B), a finding consistent with fecal excretion as the main route of $\mathrm{Mn}$ excretion. ${ }^{54} \mathrm{Mn}$ levels were reduced in carcass and nearly all tissues and increased in the GI contents of mice raised on a high-Mn diet (Figure 4, C-E).

Since Mn excretion is largely attributed to hepatobiliary excretion, we next assessed biliary Mn levels in WT mice on a control or high-Mn diet using 2 approaches. First, prior to tissue harvesting from the mice described above, we collected bile for 1 hour from anesthetized mice by ligation of the common bile duct proximal to the pancreas and cannulation of the gallbladder (see Supplemental Video 1). Biliary ${ }^{54} \mathrm{Mn}$ levels were elevated in WT mice on 
A

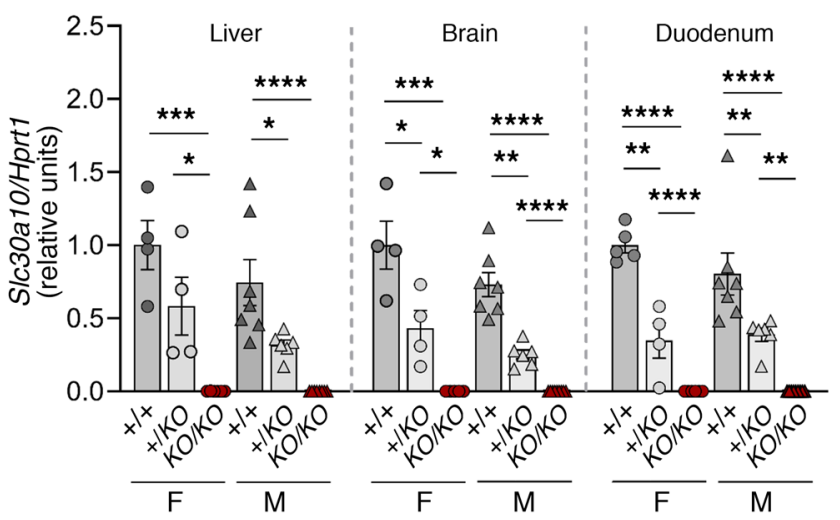

B

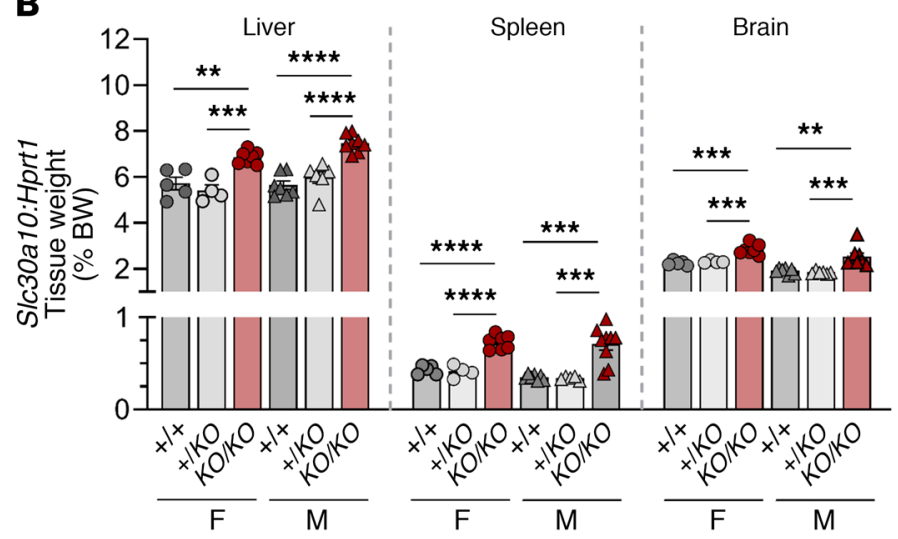

C

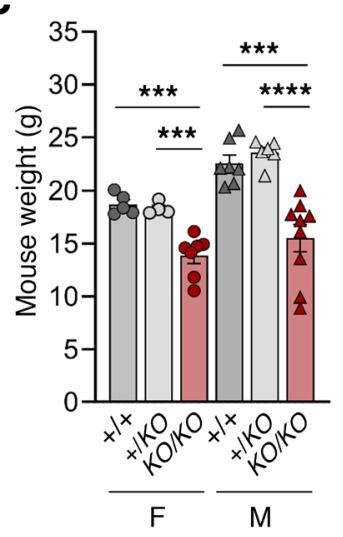

D

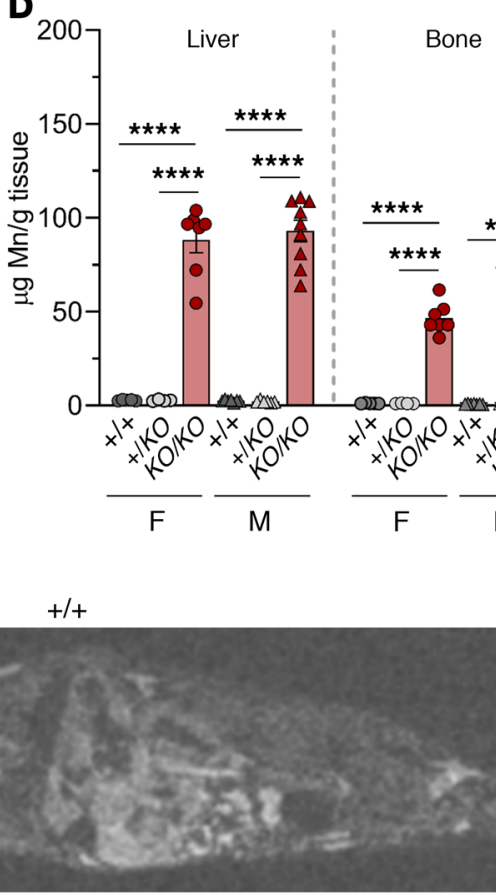

Bone

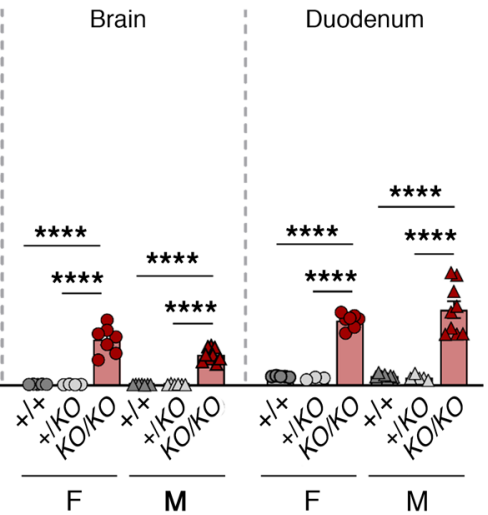

$\mathbf{E}$
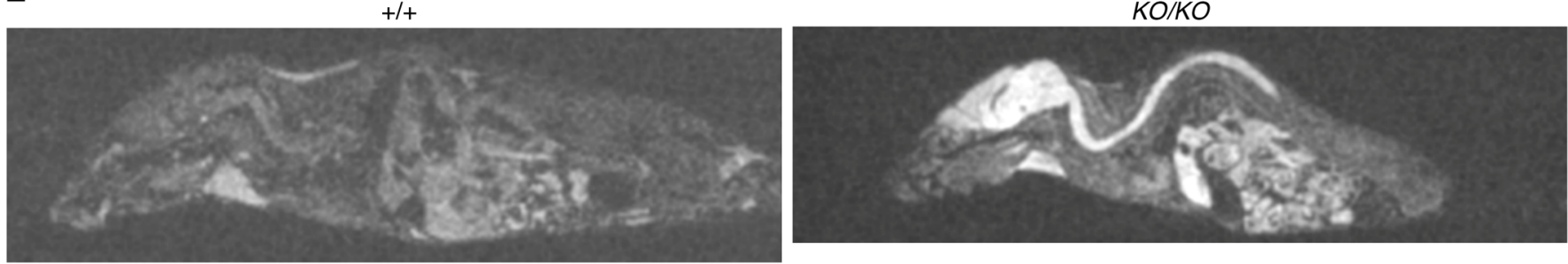

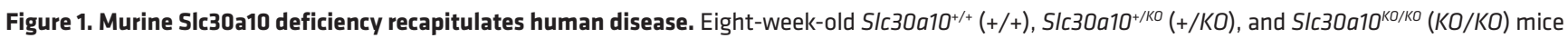
were characterized for (A) tissue SIc30a10/Hprt1 RNA ratios, normalized to levels in SIc30a10 +/+ females; (B) tissue weights as a percentage of body weight; (C) body weight; and (D) tissue Mn levels. (E) MRIs (sagittal views). For A-D, $P$ values were calculated by 1-way ANOVA with Tukey's multiple comparisons test. Data are presented as individual values and represent the mean \pm SEM. Outliers (not shown) were identified by the ROUT method. Removal of outliers in $\mathbf{A}$ did not alter the identification of comparisons with a $P$ value below 0.05 . No outliers were identified in $\mathbf{C}$ or $\mathbf{D}$. Removal of the outlier for male $K O / K O$ liver (4.180\%) in B decreased the $P$ value versus $+/ K O$ from $P>0.05$ to $P<0.0001$. For all panels, $n=5-9$ replicates/group, except for female $S / c 30 a 10^{+/ K O}$ mice $(n=3-4) .{ }^{*} P<0.05,{ }^{* *} P<0.01,{ }^{* *} P<0.001$, and ${ }^{* * *} P<0.0001$. F, female; $M$, male.

a high-Mn diet (Figure 4F). Second, a separate set of mice were subjected to collection of nonradioactive bile followed by measurement of total biliary metal levels. In accordance with ${ }^{54} \mathrm{Mn}$ levels, we observed that biliary Mn levels were increased in WT mice raised on a high-Mn diet (Figure 4G). Although copper is also excreted through the hepatobiliary system (33), biliary copper levels were not affected by diet in female mice but were decreased in male mice raised on a high-Mn diet (Figure $4 \mathrm{H}$ ). During bile collections, we also assessed bile flow rates and found that the flow rates were decreased in male but not female mice raised on a highMn diet (Figure 4I). The significance of this is not clear, but it is unlikely that decreased bile volumes contributed to the increased biliary Mn levels observed with Mn excess.
Slc30a10 is essential for systemic and hepatobiliary $\mathrm{Mn}$ excretion. To understand the role of Slc30a10 in systemic and hepatobiliary Mn excretion, we next assessed Mn excretion in Slc3Oa1O $\mathrm{O}^{+/+}$and Slc3Oa1O $\mathrm{KO} / \mathrm{KO}$ mice. To evaluate systemic Mn excretion, mice were retro-orbitally injected with ${ }^{54} \mathrm{Mn}$ and housed overnight in metabolic cages for collection of urine and feces. In contrast to increased fecal ${ }^{54} \mathrm{Mn}$ levels in WT mice raised on a high-Mn diet (Figure 4A), we observed that fecal

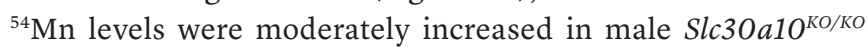

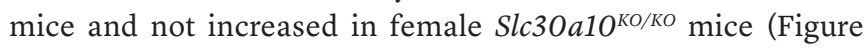
$5 \mathrm{~A})$. Given the severe levels of tissue Mn excess in $\mathrm{Slc30a1O^{KO/KO }}$ mice, these ${ }^{54} \mathrm{Mn}$ fecal levels were inappropriately low, suggesting impaired systemic excretion. Urinary ${ }^{54} \mathrm{Mn}$ levels 
A

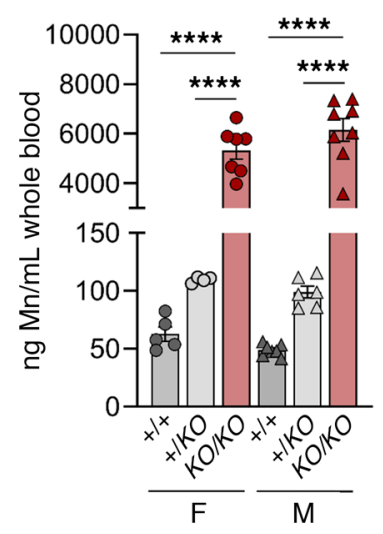

E

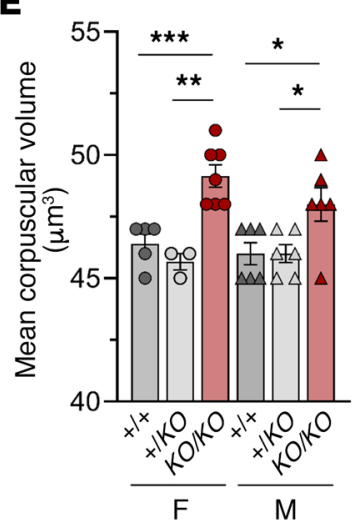

B
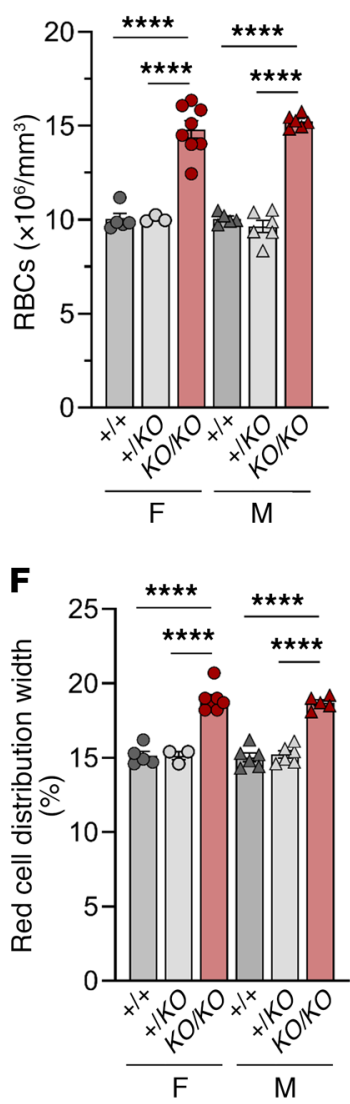

C

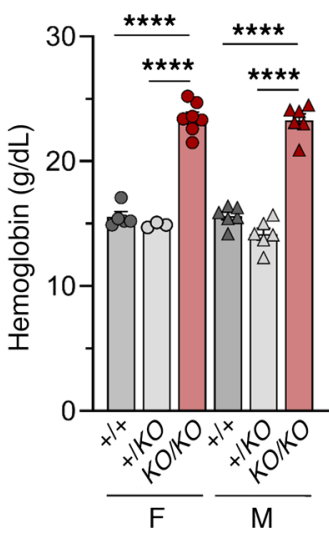

G

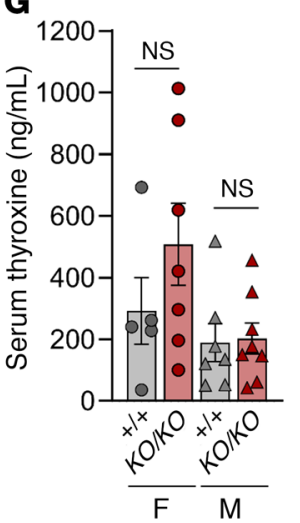

D

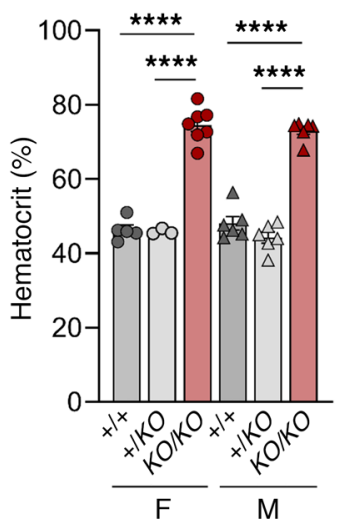

H

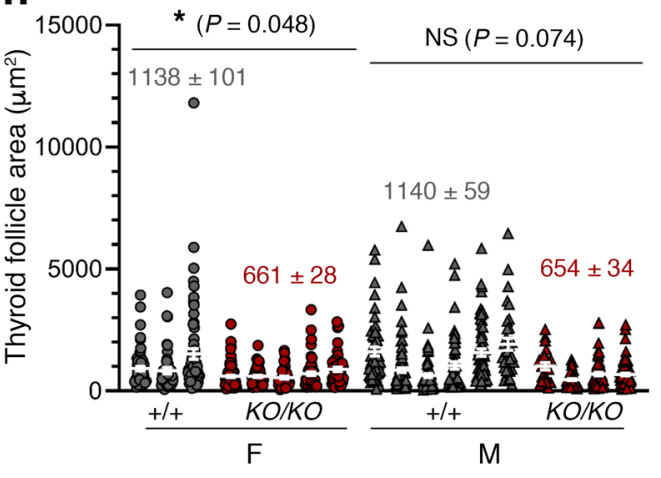

I
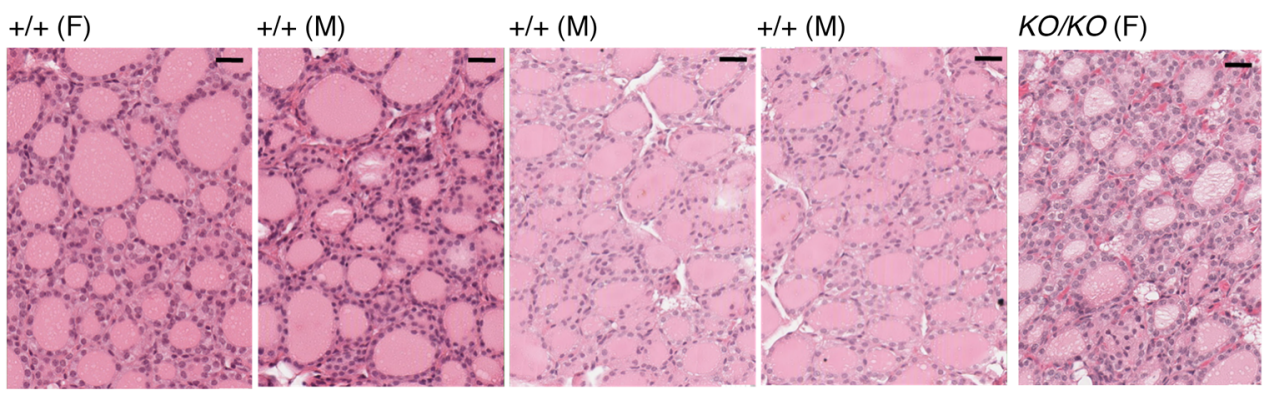

$K O / K O(F)$

$\mathrm{KO} / \mathrm{KO}(\mathrm{M})$

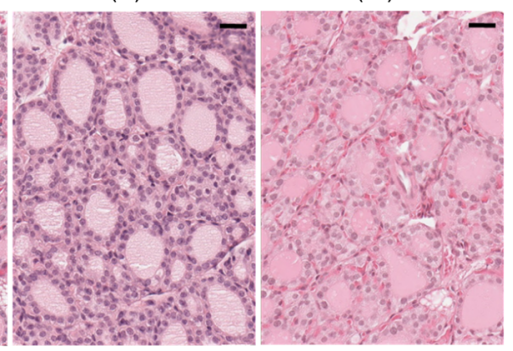

Figure 2. SIc30a10-deficient mice have increased RBC parameters and mild thyroid pathology. Eight-week-old S/c30a10+/+, S/c30a10+/Ko, and S/c30a10 $\mathrm{KO} / \mathrm{KO}^{\circ}$ mice were characterized for (A) blood Mn levels; (B) RBC counts; (C) hemoglobin levels; (D) hematocrit levels; (E) mean corpuscular volumes; (F) red cell distribution widths; and (C) thyroxine (T4) levels. For A-G, $P$ values were calculated by 1-way ANOVA with Tukey's multiple comparisons test. No outliers were identified by ROUT. Data are presented as individual values and represent the mean \pm SEM. $n=5-9$ replicates/group, except for female S/c30a10+/KO mice $(n=3-4) .{ }^{*} P<0.05,{ }^{* *} P<0.01,{ }^{* *} P<0.001$, and ${ }^{* * *} P<0.0001$. $(\mathbf{H})$ Thyroid follicle area. Each vertical group represents 1 mouse, with each point representing 1 follicle ( $n=21-96$ follicles/mouse). The mean \pm SEM for each mouse is shown with white bars and lines. The numbers above the groups represent the mean $\pm \mathrm{SEM}$ for each sex and genotype. Two-tailed $P$ values were calculated by nested $t$ test. (I) H\&E-stained images of thyroid. Original magnification, $\times 40$; scale bars: $25 \mu \mathrm{m}$.

were minimal compared with fecal ${ }^{54} \mathrm{Mn}$ levels but were increased in male $\mathrm{Slc} 30 \mathrm{a} 1 \mathrm{O}^{\mathrm{KO} / \mathrm{KO}}$ mice (Figure 5B). A similar increase in urinary ${ }^{54} \mathrm{Mn}$ levels has been shown in Slc39a14deficient mice, another model of inherited Mn excess (18).

To assess biliary $\mathrm{Mn}$ and copper levels, bile was collected for 1 hour following bile duct ligation and gallbladder cannulation in

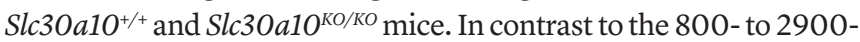
fold difference in biliary Mn levels between WT mice raised on a control or high-Mn diet (Figure 4G), biliary Mn levels were similar in
Slc3Oa1O+/+ and Slc30a1O KO/KO mice (Figure 5C). When normalized to total liver Mn levels, biliary Mn levels were lower in Slc3Oa1O ${ }^{K O / K O}$ mice, whereas biliary Mn levels in WT mice raised on a high-Mn diet remained higher than in mice raised on a control Mn diet (Figure 5D). Female Slc30a10 ${ }^{K O / K O}$ mice had lower biliary copper levels than did Slc3Oa1O ${ }^{+/+}$mice (Figure 5E), a phenomenon we observed in Mnloaded rats (34). Together, these results indicate that biliary $\mathrm{Mn}$ levels in Slc30a1OKO/KO mice were inappropriately low, given the level of Mn excess in these mice. 

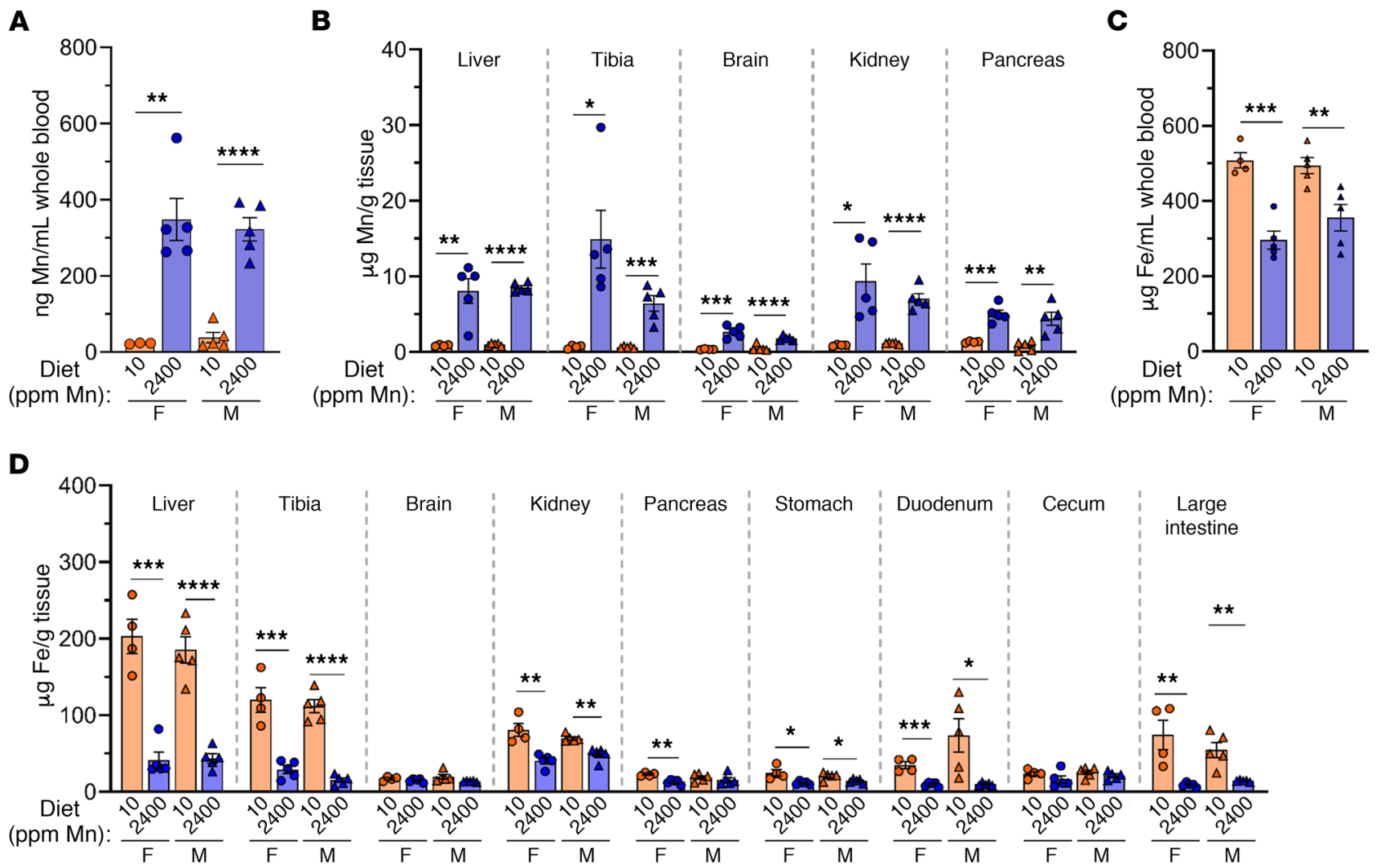

E

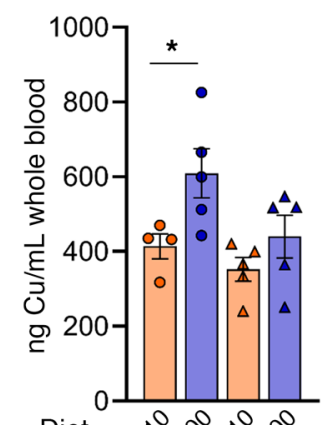

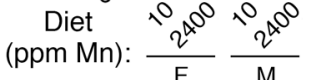

$\mathbf{F}$

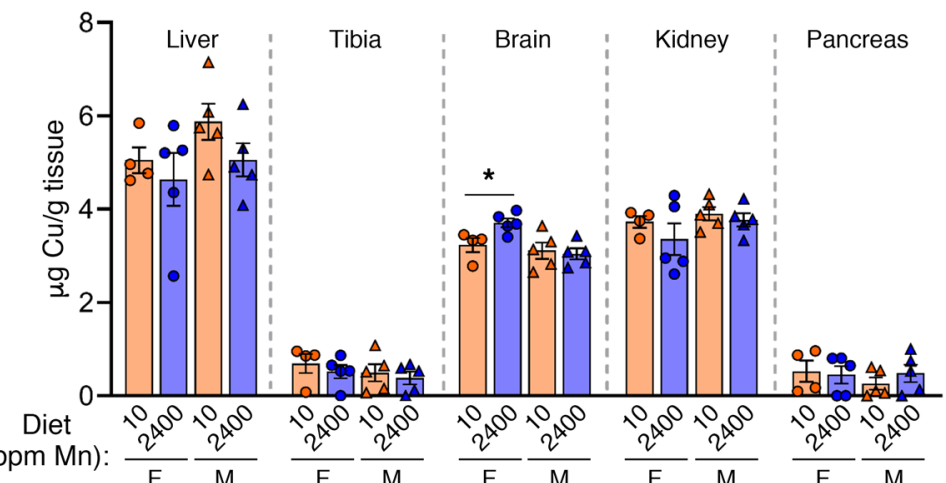

G

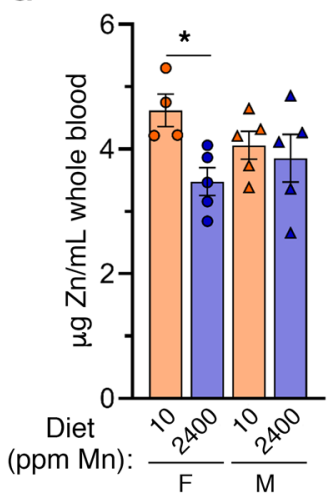

H

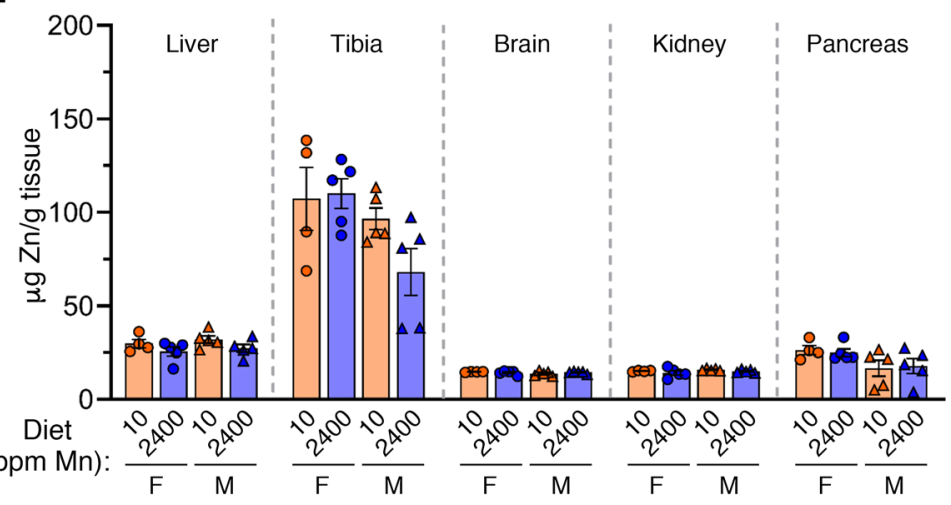

Figure 3. Tissue metal levels in 8-week-old WT mice raised on a control or high-Mn diet. C57BL/6N) mice weaned onto a control Mn (10 ppm) or high-Mn (2400 $\mathrm{ppm}$ ) diet were characterized at 8 weeks of age for blood $(\mathbf{A}, \mathbf{C}, \mathbf{E}$, and $\mathbf{G})$ and tissue $(\mathbf{B}, \mathbf{D}, \mathbf{F}$, and $\mathbf{H})$ levels of $\mathrm{Mn}(\mathbf{A}$ and $\mathbf{B})$, iron $(\mathrm{Fe})(\mathbf{C}$ and $\mathbf{D})$, copper $(\mathbf{C u})(\mathbf{E}$ and $\mathbf{F})$, and zinc $(\mathrm{Zn})(\mathbf{G}$ and $\mathbf{H})$. Data are presented as individual values and represent the mean \pm SEM. Outliers (not shown) were identified by ROUT. $P$ values were calculated by unpaired, 2-tailed $t$ test. Removal of the outliers in $\mathbf{A}$ and $\mathbf{B}$ did not alter the identification of comparisons with $P$ values below 0.05 . No outliers were identified in C-H. $n=5$ replicates/group, except for females on a $10 \mathrm{ppm}$ diet $(n=4-5) .{ }^{*} P<0.05,{ }^{* *} P<0.01,{ }^{* * *} P<0.001$, and ${ }^{* * * *} P<0.0001$. 
A

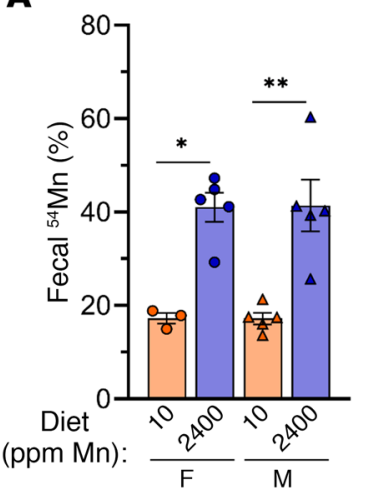

B

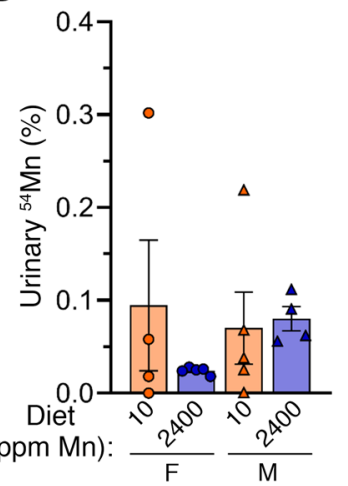

C

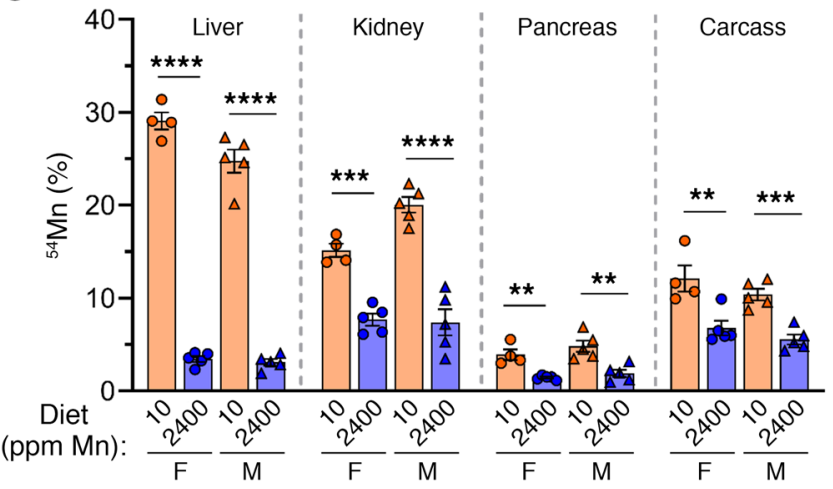

D

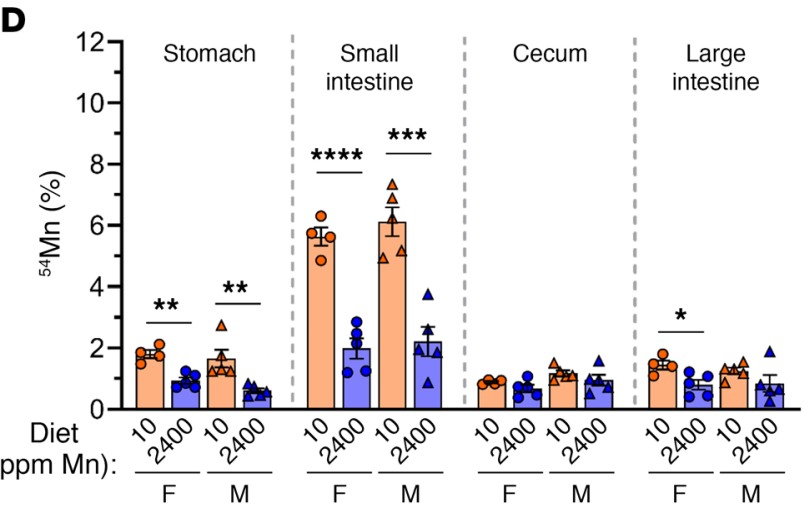

E

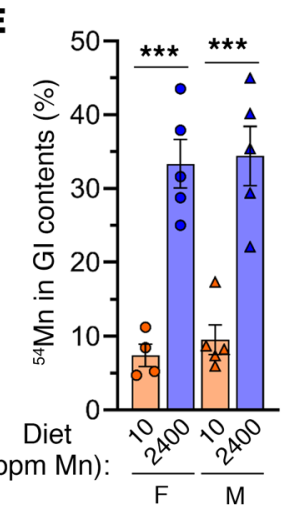

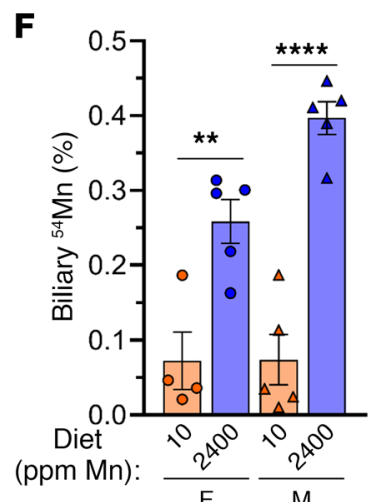
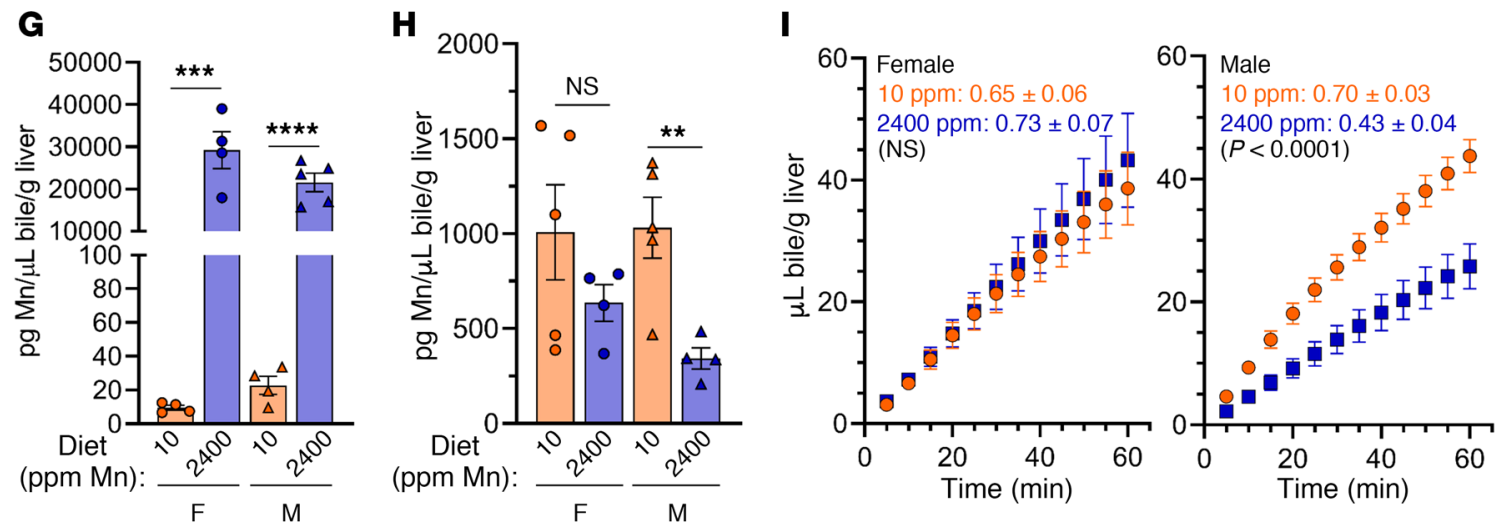

Figure 4. Hepatobiliary Mn excretion is increased in WT mice raised on a high-Mn diet. C57BL/6N) mice weaned onto a control Mn (10 ppm) or high-Mn $(2400 \mathrm{ppm})$ diet were characterized at 8 weeks of age. (A-D) ${ }^{54} \mathrm{Mn}$ levels in feces (A), urine (B), tissues/compartments (C-E), and bile (F) after ${ }^{54} \mathrm{Mn}$ injection and overnight housing in metabolic cages, presented as a percentage of total ${ }^{54} \mathrm{Mn}$ levels. (G and $\mathbf{H}$ ) Total biliary $\mathrm{Mn}(\mathbf{C})$ and copper (H) levels. For $\mathbf{A}-\mathbf{H}$, data are presented as individual values and represent the mean \pm SEM. Two-tailed $P$ values were calculated by unpaired $t$ test. Outliers (not shown) were identified by ROUT. No outliers were identified in $\mathbf{A}-\mathbf{F}$ or $\mathbf{H}$. Removal of the outliers in $\mathbf{G}$ did not alter the identification of comparisons with $P$ values below 0.05 . (I) Bile flow rates following bile duct ligation and gallbladder cannulation in female (left) and male (right) mice. Each data point represents the mean \pm SEM. The values shown in I indicate the average slope of the line \pm SEM for each group followed by the $P$ value for comparison of line slopes by linear regression. $n=5$ replicates/group except for females on a control Mn diet $(n=4-5)$. ${ }^{*} P<0.05,{ }^{*} P<0.01,{ }^{* * *} P<0.001$, and ${ }^{* * * *} P<0.0001$.

To further explore the effect of Slc30a10 deficiency on biliary Mn excretion, we next assessed the rate of bile synthesis and ${ }^{54} \mathrm{Mn}$ export into bile in $\mathrm{Slc} \mathrm{Oa} \mathrm{alO}^{+/+}$and $\mathrm{Slc} 3 \mathrm{Oa} 1 \mathrm{O}^{\mathrm{KO} / \mathrm{KO}}$ mice using the surgical approach described above, with one difference. Immediately after bile duct ligation and gallbladder cannulation, we injected ${ }^{54} \mathrm{Mn}$ into the portal vein. We found that blood ${ }^{54} \mathrm{Mn}$ levels were minimal and liver ${ }^{54} \mathrm{Mn}$ uptake was unimpaired, indicating intact $\mathrm{Mn}$ uptake into liver parenchyma (Figure $5 \mathrm{~F}$ ). Bile flow rates were similar in female $\mathrm{Slc}_{\mathrm{O}} \mathrm{Oa1O^{+/+ }}$ and $\mathrm{Slc} \mathrm{Oa} 1 \mathrm{O}^{\mathrm{KO} / \mathrm{KO}}$ mice but were decreased in male Slc30a10 KO/KO mice (Figure 5G). This differed from our dietary studies, in which male mice raised on a high-Mn diet had decreased bile flow rates (Figure 4I). (The significance of this is unclear.) Biliary ${ }^{54} \mathrm{Mn}$ levels were undetectable

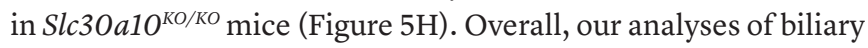
Mn excretion using nonradioactive and radioactive approaches indicated that Slc30a10 is essential for Mn export into bile.

Hepatocyte Slc30a1O deficiency does not recapitulate disease phenotypes. To further explore the role of Slc30a10 in hepatobi- 
A

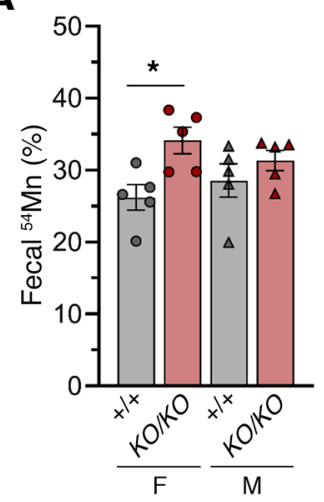

E

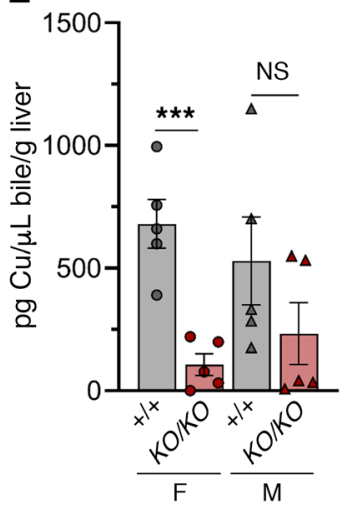

H

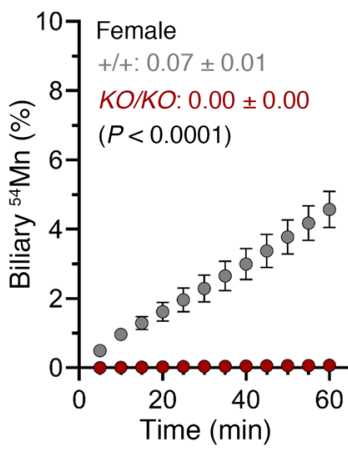

B

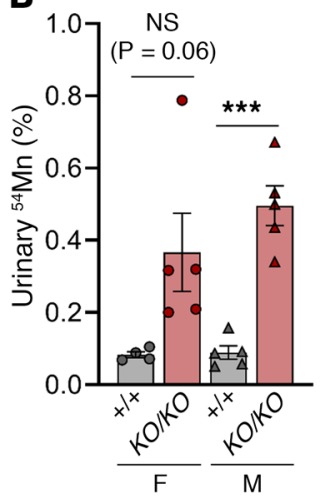

C

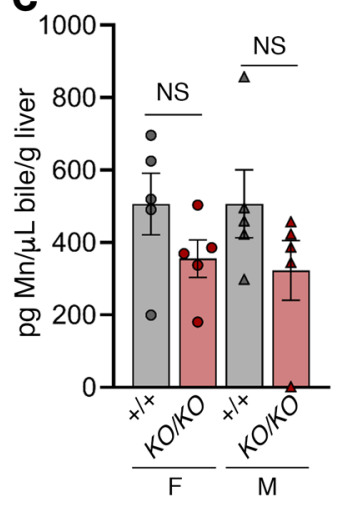

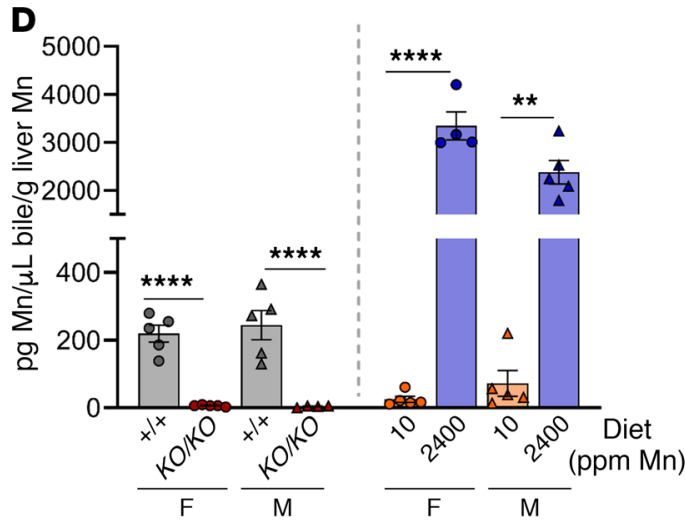

$\mathbf{F}$

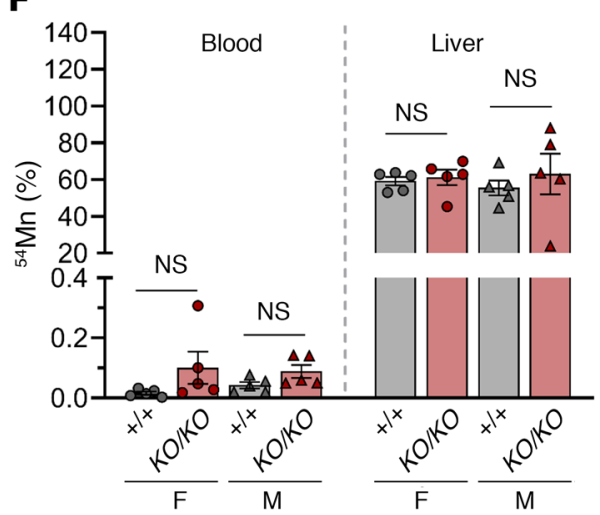

G

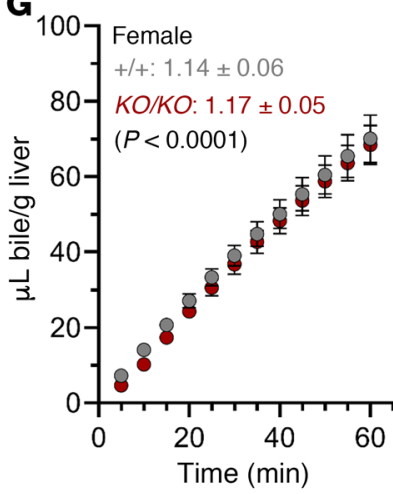

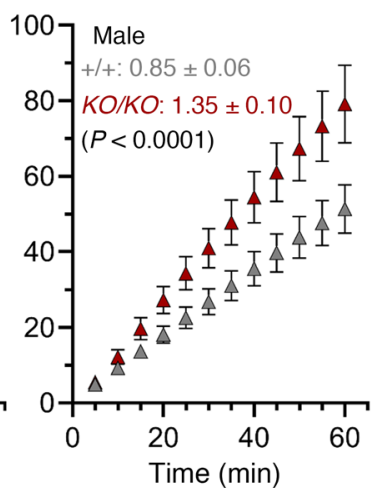

Figure 5. SIc30a10 is essential for systemic and hepatobiliary Mn excretion. Eightweek-old Slc30a10+/+ and S/c30a10 KO/KO mice were characterized for (A and B) fecal (A) and urinary (B) ${ }^{54} \mathrm{Mn}$ levels after retro-orbital ${ }^{54} \mathrm{Mn}$ injection and overnight housing in metabolic cages (data are presented as a percentage of total ${ }^{54} \mathrm{Mn}$ ); (C and D) biliary Mn levels normalized to liver weight (C) and Mn content (D) (in D, biliary Mn levels from WT mice on a control or high-Mn diet are included for reference); and (E) biliary copper levels. (F-H) Mice underwent bile duct ligation, gallbladder cannulation, ${ }^{54} \mathrm{Mn} /$ fluorescein injection into the portal vein, and bile collection for 2 hours. (F) Blood and liver ${ }^{54} \mathrm{Mn}$ levels. (G and $\mathbf{H}$ ) Bile volume (G) and biliary ${ }^{54} \mathrm{Mn}$ levels (H) versus time for female (left) and male (right) mice. Each data point represents the mean \pm SEM. Values shown in $\mathbf{G}$ and $\mathbf{H}$ indicate the average slope of the line \pm SEM for each group followed by a $P$ value for comparison of line slopes by linear regression. In A-F, data are presented as individual values and represent the mean \pm SEM. Outliers (not shown) were identified by ROUT. Two-tailed $P$ values were calculated by unpaired $t$ test. No outliers were identified in A-C, E, or F. Removal of the outlier in $\mathbf{D}$ did not alter the identification of comparisons with a $P$ value below 0.05 . For all panels, $n=5$ replicates/group, except for females on a high-Mn diet $(n=4)$. ${ }^{*} P<$ $0.05,{ }^{* *} P<0.01,{ }^{* *} P<0.001$, and ${ }^{* * *} P<0.0001$.

liary excretion, we next identified hepatic cell types that express Slc30a10. Slc30a10 RNA is abundantly expressed only in parenchymal cells (Figure 6A). As commercial antibodies do not reliably detect endogenous mouse Slc30a10 and our attempts at producing anti-Slc30a10 antibodies were unsuccessful (data not shown), we used CRISPR/Cas9 to introduce a GFP cDNA immediately upstream of the stop codon of the Slc30a10 gene (Slc30a10 ${ }^{\text {GFP/GFP }}$ ) (Supplemental Figure 1F). We found that tissue Mn levels were similar in $\mathrm{Slc3Oa1O^{+/+ }}$ and Slc3Oa1O ${ }^{\text {GFP/GFP }}$ mice but were slightly increased in Slc3Oa1O ${ }^{K O / G F P}$ mice, suggesting that the GFP allele is mildly hypomorphic (Figure 6B). In Slc30a1O ${ }^{\text {GFP/GFP }}$ mice, liver fluorescence was most prominent in hepatocyte membranes, and the fluorescence pattern resembled staining with antibodies against
MDR1, a canalicular membrane marker (Figure 6, C and D). This result is similar to that previously reported in cell lines (35).

Since Slc30a10 is expressed in hepatocytes and is essential for biliary Mn excretion, we hypothesized that hepatocyte Slc30a10 is essential for Mn excretion. To this end, we generated mice with hepatocyte Slc30a10 deficiency using an albumin promoter-driven Cre recombinase transgene $\left(\mathrm{Slc30a1O^{lox } / l o x} \mathrm{Alb}\right)$ (Supplemental Figure $1 C$ ). We first characterized the effect of the $S l c 30 a 1 O^{\text {lox }}$ allele and $A l b$ transgene on Mn levels. Tissue Mn levels were largely similar between $\mathrm{Slc}_{\mathrm{O} O a 1 \mathrm{O}^{+/+}}$and Slc3Oa1O lox/lox mice, and tissue Mn levels did not differ between $\mathrm{Slc3Oa1O}^{+/+}$and $\mathrm{Slc3Oa1O}^{+/+} \mathrm{Alb}$ mice (Supplemental Figure 6, A-C). We next assessed Slc30a1O

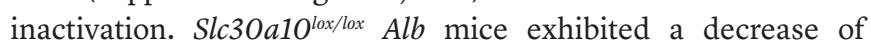


A

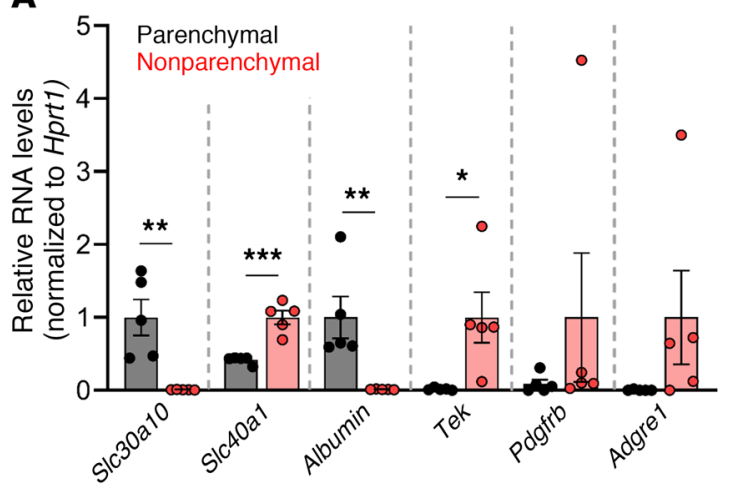

C

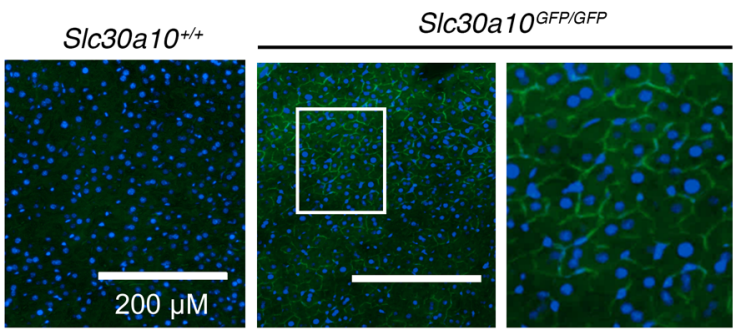

B

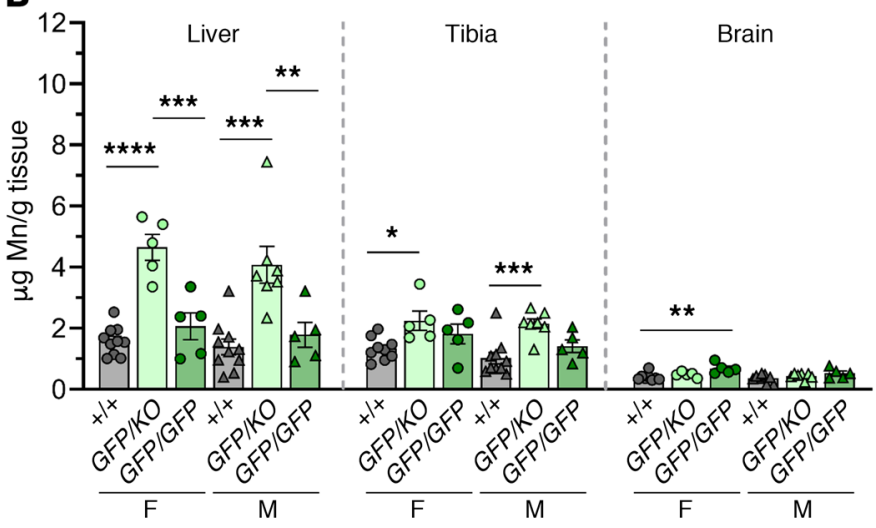

D

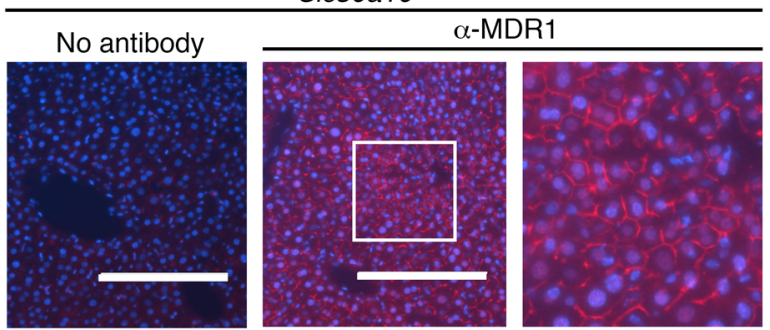

Figure 6. SIc30a10 is localized to the canalicular hepatocyte membrane. (A) RNA levels of transporters and cell type-specific markers relative to Hprt1 levels: albumin (hepatocytes), Tek (sinusoidal endothelial cells), Pdgfrb (stellate cells), and Adgre1 (Kupffer cells). For each gene, the cell type with higher expression

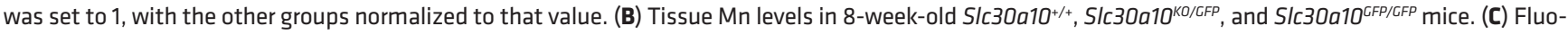
rescence images of 2-month-old SIc30a10+/+ and SIc30a10 GFP/GFP frozen liver sections. DAPI (blue); GFP (green). Original magnification, $\times 20 ;$ scale bars: $200 \mu \mathrm{m}$; original magnification, $\times 50$ (enlarged inset). (D) Immunofluorescence images of frozen liver sections from 2-month-old SIc30a10 ${ }^{\text {CFP/CFP }}$ mice. DAPI (blue); anti-MDR1 ( $\alpha$-MDR1) (red). Original magnification, $\times 20$ and $\times 50$ (enlarged inset); scale bars: $200 \mu \mathrm{m}$. In A and B, data are presented as individual values and represent the mean \pm SEM. In A, 2-tailed $P$ values were calculated by unpaired $t$ test. $n=5$ replicates/group. In B, removal of the outliers identified by the

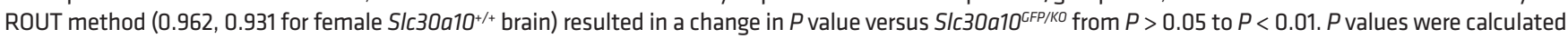
by 1-way ANOVA with Tukey's multiple comparisons test. $n=5-10$ replicates/group. ${ }^{*} P<0.05,{ }^{* *} P<0.01,{ }^{* * *} P<0.001$, and ${ }^{* * * *} P<0.0001$.

approximately $90 \%$ in liver Slc30a10 RNA levels at 3 weeks of

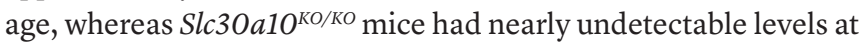
the same age (Supplemental Figure 6D). Given this and a preliminary analysis indicating a lack of Mn excess in 2-month-old mice (data not shown), we characterized $S l c 30 a 10^{l o x} / l o x$ Alb mice at 4 months of age. Slc30a1O ${ }^{l o x} /$ lox $\mathrm{Alb}$ mice had liver-specific Slc30a10 deficiency but only an approximately 2-fold increase in liver Mn levels and lesser increases in Mn levels in male bone and brain (Figure 7, A and B), in contrast to the severe Mn excess seen in Slc30 a1O ${ }^{\mathrm{KO} / \mathrm{KO}}$ mice. Additionally, RBC levels, body size, and organ

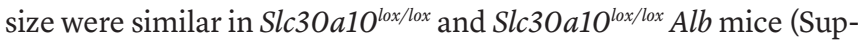
plemental Figure 6E and data not shown). Overall, these data indicated that Slc30a10 expression in hepatocytes of adult mice is not essential for systemic Mn homeostasis. A more prominent phenotype may have been observed in $S l c 30 a 10^{l o x / l o x} \mathrm{Alb}$ mice if the $\mathrm{Alb}$ transgene inactivated Slc30a10 earlier in development.

Hepatocyte Slc4Oa1 does not compensate for hepatocyte Slc30a1O deficiency. Besides Slc30a10, Slc40a1 (ferroportin) is the only other transporter implicated in cellular Mn export in vivo $(8,13,14)$. Since Slc40a1 is expressed on the basolateral hepatocyte membrane (36), we hypothesized that hepatocyte Slc40a1 could com-

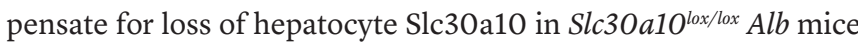
by exporting $\mathrm{Mn}$ from the liver into blood for excretion from routes independent of the hepatobiliary system. To test this, we generated and characterized mice deficient in hepatocyte Slc30a10 and

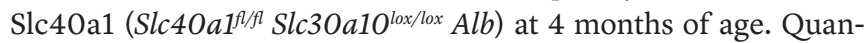
titative PCR (qPCR) confirmed liver-specific Slc30a1O deficiency and decreased Slc40a1 expression (Figure 7, C and D). The retained Slc40a1 expression in liver most likely reflects expression in nonparenchymal cells (Figure 6A). Body weight and blood

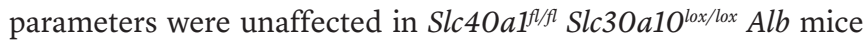
(data not shown). We observed minimal Mn excess in mutant mice bone (Figure 7E). Liver and female bone iron levels were slightly

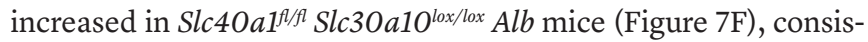
tent with previous reports of aberrant iron levels in mice with liver Slc40a1 deficiency (37). Overall, these data indicated that hepatocyte Slc40a1 was not compensating for hepatocyte Slc30a10 deficiency and that long-term hepatocyte-specific deficiency of both proteins results in only minimal Mn excess.

Hepatocyte Slc30a10 is required for biliary Mn excretion. To further explore the minimal Slc30a10 lox/lox Alb phenotype, we assessed systemic Mn excretion in Slc3Oa1O lox/lox and Slc30a1O ${ }^{\text {lox/lox }}$ $A l b$ mice. Mice were retro-orbitally injected with ${ }^{54} \mathrm{Mn}$ and housed in metabolic cages overnight. ${ }^{54} \mathrm{Mn}$ was predominantly excreted via feces, and we observed no differences in fecal or urinary ${ }^{54} \mathrm{Mn}$

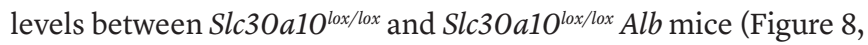
$A$ and B). Prior to tissue harvesting, the mice were subjected to bile collection via gallbladder cannulation. Although liver ${ }^{54} \mathrm{Mn}$ levels 
A

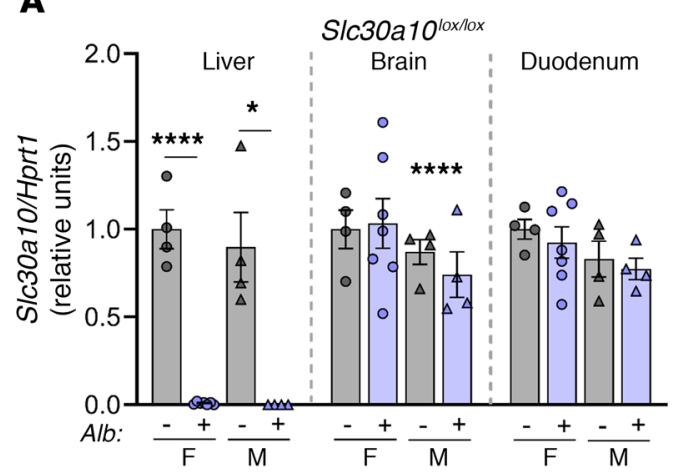

C

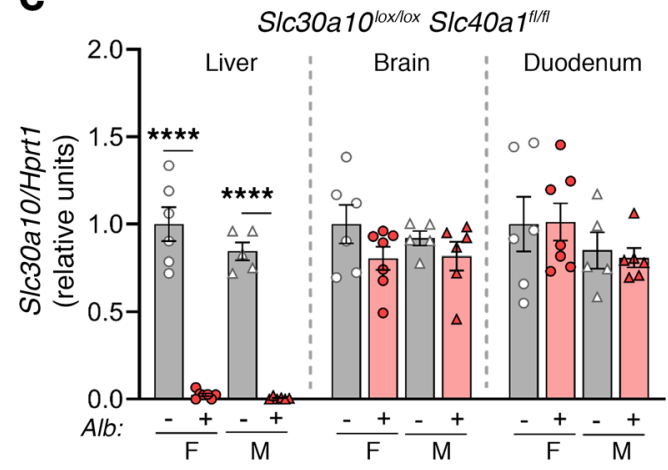

E

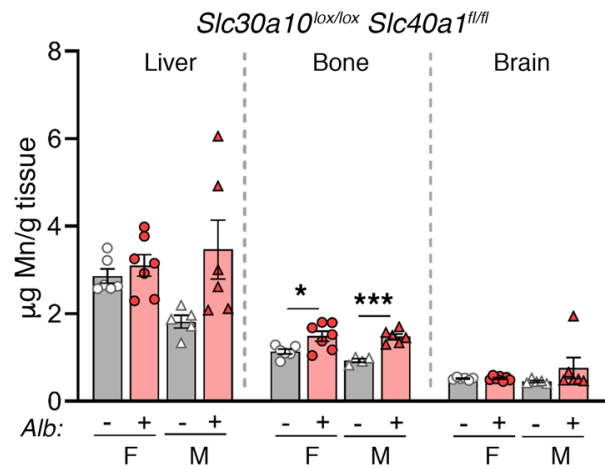

B

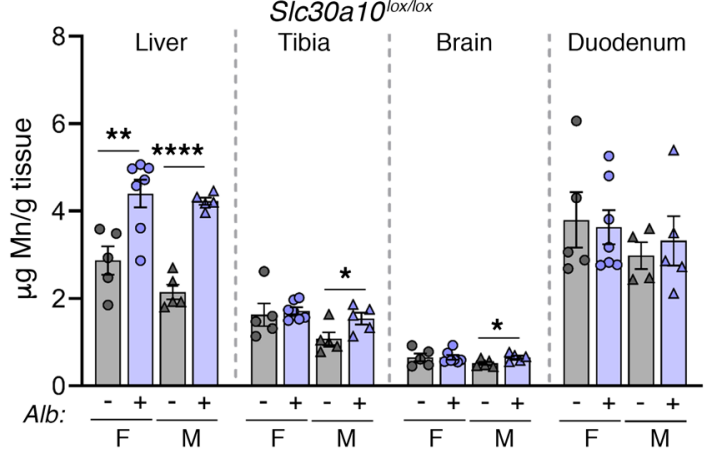

D

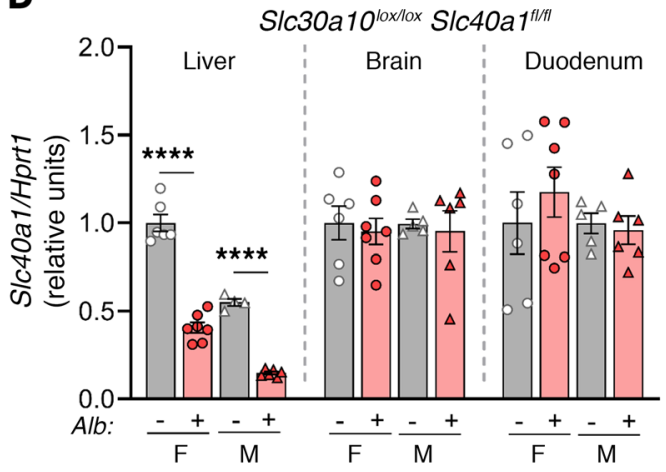

$\mathbf{F}$

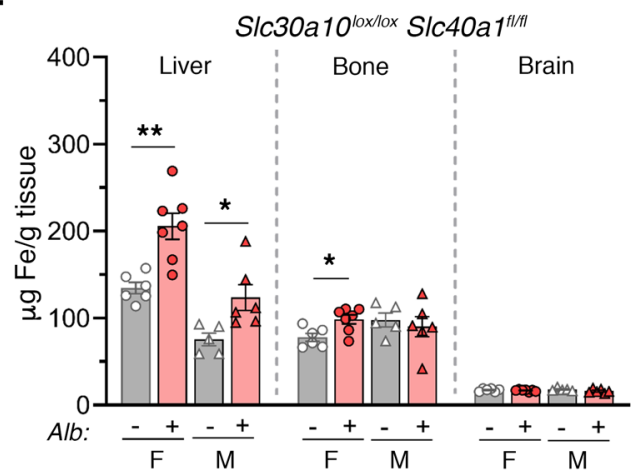

Figure 7. Hepatocyte SIc30a10 deficiency, irrespective of hepatocyte Slc40a1 deficiency, leads to minimal Mn excess. (A and B) Four-month-old

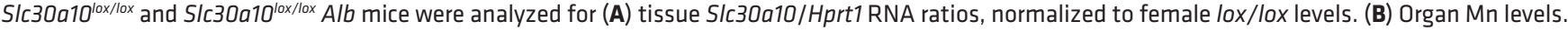

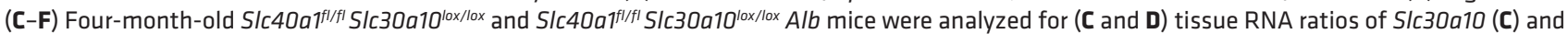
SIc40a1 (D) to Hprt1, normalized to female fl/fl lox/lox levels, and (E and F) organ Mn (E) and iron (F) levels. In all panels, data are presented as individual values and represent the mean \pm SEM. Outliers (not shown) were identified by ROUT. Two-tailed $P$ values were calculated by unpaired $t$ test. Removal of the outliers in $\mathbf{A}, \mathbf{B}$, and $\mathbf{D}$ did not alter the identification of comparisons with a $P$ value below 0.05 . No outliers were identified in $\mathbf{C}$ or $\mathbf{F}$. Removal of the outlier in E (4.351 for male SIc30a10 ${ }^{+/+}$brain) changed the $P$ value from $P>0.05$ to $P<0.001 . n=5-7$ replicates/group, except for female S/c30a10 $0^{10 x / 10 x}$, male Slc30a10 ${ }^{10 x / 10 x}$, and male SIc30a10 ${ }^{10 x / 10 x} \mathrm{Alb}(n=4-5)$ mice. ${ }^{*} P<0.05,{ }^{* *} P<0.01,{ }^{* * *} P<0.001$, and ${ }^{* * * *} P<0.0001$.

were increased in $S l c 30 a 10^{l o x} / l o x$ Alb mice, biliary ${ }^{54} \mathrm{Mn}$ counts were nearly undetectable in Slc30a10 ${ }^{l o x} /$ lox $A l b$ mice (Figure 8C). Total biliary $\mathrm{Mn}$ levels were minimal and copper levels unchanged in Slc30a10 ${ }^{b x / l o x} \mathrm{Alb}$ mice (Figure 8D), in contrast to the similar biliary $\mathrm{Mn}$ and reduced copper levels detected in Slc3Oa10 KO/KO mice (Figure 5, C and E). To determine whether hepatic Slc30a10 deficiency affected hepatobiliary Mn excretion, we next assessed bile flow rates and ${ }^{54} \mathrm{Mn}$ export into bile. Although the bile flow rates

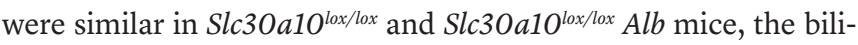
ary ${ }^{54} \mathrm{Mn}$ levels were minimal in mutant mice (Figure 8, $\mathrm{E}$ and $\mathrm{F}$ ). Overall, these data indicated that hepatocyte Slc30a10 is essential for biliary Mn excretion but dispensable for systemic Mn homeostasis in adult mice. Taken together with the severe Mn excess seen in $S 1 c 30 a 10^{K O / K O}$ mice, these data also suggested that other sites of Slc30a10 expression may compensate for impaired hepatobiliary Mn excretion.

Enterocyte Slc30a10 contributes to the regulation of tissue $\mathrm{Mn}$ levels. qPCR revealed abundant Slc30a10 RNA expression in the GI tract, with the highest expression detected in the duodenum (Figure 9A). Slc30a10 ${ }^{\text {GFP } / G P P}$ mice exhibited fluorescence on the apical surface of duodenal enterocytes and cecal epithelium (Figure 9B) and sporadically on the apical surface of large intestine 
A

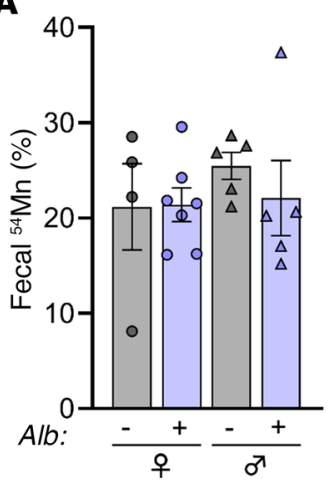

D
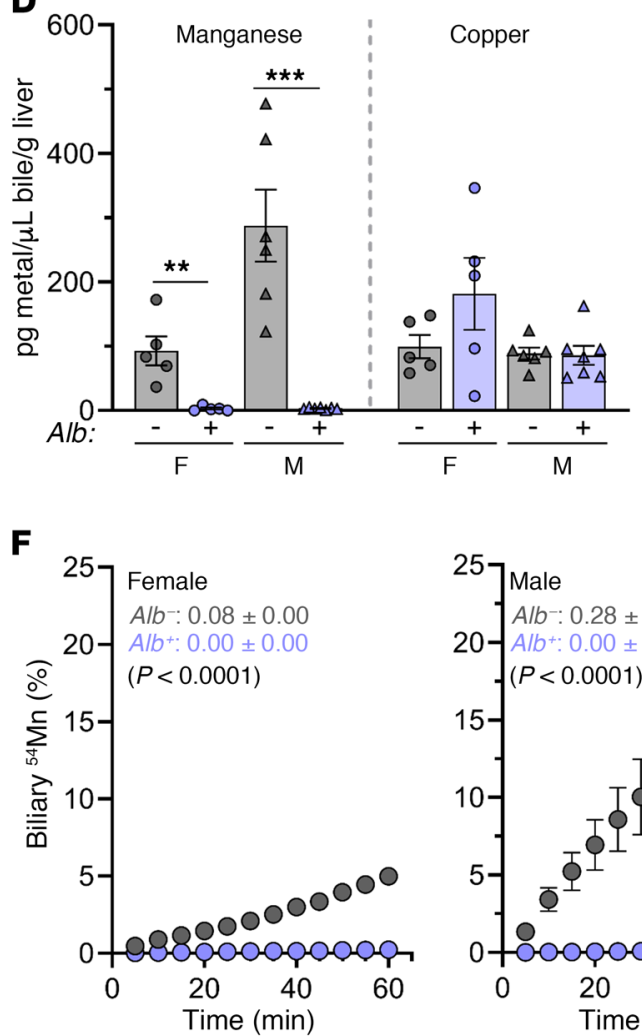

B

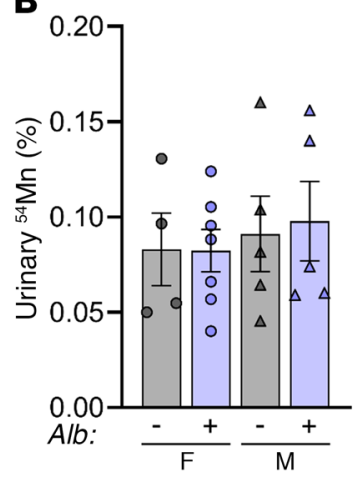

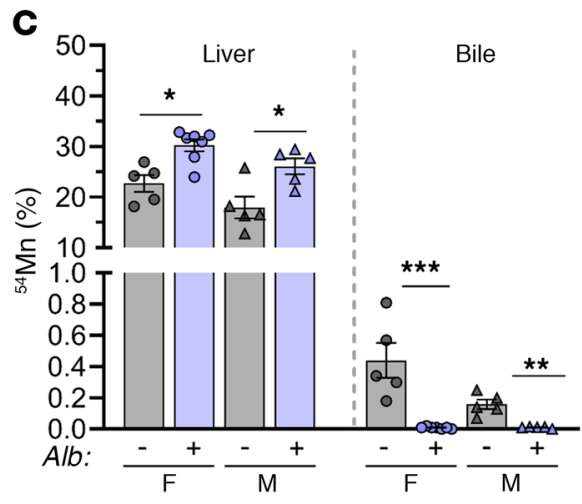

E

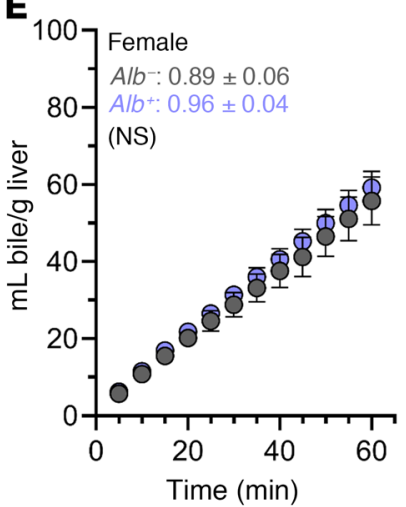

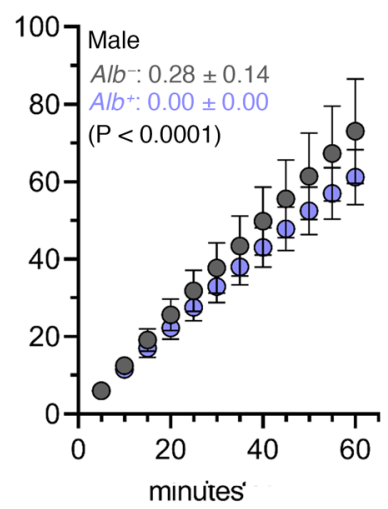

Figure 8. Hepatic SIc30a10 is essential for hepatobiliary Mn excretion. Four-

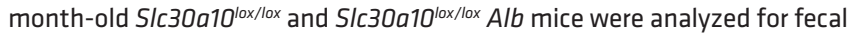
(A), urinary (B), and liver and bile (C) ${ }^{54} \mathrm{Mn}$ levels after ${ }^{54} \mathrm{Mn}$ injection, overnight housing in metabolic cages, gallbladder cannulation, and 1-hour bile collection. Levels are expressed as a percentage of total ${ }^{54} \mathrm{Mn}$ levels. (D) Total biliary $\mathrm{Mn}$ and copper levels, normalized to liver mass. (E and F) Another set of female (left) and male (right) mice underwent bile duct ligation, gallbladder cannulation, ${ }^{54} \mathrm{Mn}$ /fluorescein injection via the portal vein, and 1-hour bile collection. (E) Biliary volumes. (F) Bile ${ }^{54} \mathrm{Mn}$ levels. In A-D, data are presented as individual values and represent the mean \pm SEM. Outliers (data not shown) were identified by ROUT. Two-tailed $P$ values were calculated by unpaired $t$ test. No outliers were detected in $\mathbf{A}-\mathbf{D}$. In $\mathbf{E}$, each data point represent the mean $\pm S E M$. Values shown in $\mathbf{F}$ indicate the average slope of the line \pm SEM for each group followed by a $P$ value for comparison of the line slopes by linear regression. For all panels, $n=4-7$ replicates/group. ${ }^{*} P<0.05,{ }^{* *} P<0.01$, and ${ }^{* * *} P<0.001$.

enterocytes (data not shown). To determine the contribution of intestinal Slc30a10 to Mn homeostasis, we used a villin promoterdriven Cre recombinase transgene to generate mice with enterocyte-specific Slc30a10 deficiency (Slc30a10 ${ }^{\text {lox } / \text { lox }} \mathrm{Vil}$ ) (Supplemental Figure 1D). At 4 months of age, we found that tissue Mn levels were largely similar between $\mathrm{Slc}_{\mathrm{O} O \mathrm{a} 1 \mathrm{O}^{+/+}}$and $\mathrm{Slc} 3 \mathrm{Oa1O^{+/+ }} \mathrm{Vilmice}$, suggesting that the Cre transgene did not impact Mn homeostasis (Supplemental Figure 6, A-C). qPCR confirmed Slc30a10 deficiency in small intestine and intact Slc30a10 expression in liver, brain, stomach, cecum, and large intestine (Figure 9C). Slc30a10 ${ }^{\text {lox/lox }}$ Vil mice had normal body weights and RBC counts (data not shown and Supplemental Figure 6E). Slc30a10 ${ }^{\text {lox/lox }}$ Vil mice had increased Mn levels in liver, bone, duodenum, and male brain (Figure 9D). Together, these data suggested that Slc30a10 in the small intestines contributes to $\mathrm{Mn}$ homeostasis.

Hepatocyte and enterocyte Slc30a1O both contribute to the regulation of tissue Mn levels. To determine whether Slc30a10 expression in small intestines was compensating for liver Slc30a10 deficiency in $S l c 30 a 1 O^{l o x} / l o x$ Alb mice, we generated mice with hepatic and

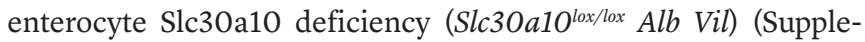
mental Figure 1E). At 4 months of age, we observed that Slc30a10 expression was deficient in liver and duodenum but retained in the brains of Slc30a10 ${ }^{b x / l o x} \mathrm{Alb}$ Vil mice (Figure 10A). Although hepatic Mn levels were lower in Slc30a10 lox/lox Alb Vil mice than in $S l c 30 a 10^{K O / K O}$ mice, duodenal Mn levels in Slc30a10 ${ }^{\text {lox } / l o x}$ Alb Vil mice matched those in Slc30a10 KO/KO mice (Figure 10B). Slc30a10 ${ }^{l o x} / 10 x$ Alb Vil mice also had increased Mn levels in other tissues and blood (Figure 10, C and D). Slc30a10 ${ }^{l o x} / / 0 x$ Alb Vil mice had normal body weights and RBC counts (data not shown and Supplemental Figure 6E). Whole blood copper levels did not differ significantly, whereas iron and zinc levels were elevated in Slc3Oa1O ${ }^{b x / l o x} \mathrm{Alb}$ Vil mice (Figure 10, E-G). These results suggested that expression of Slc30a10 in both hepatocytes and enterocytes of the small intestines contributes to the regulation of $\mathrm{Mn}$ levels. 
A

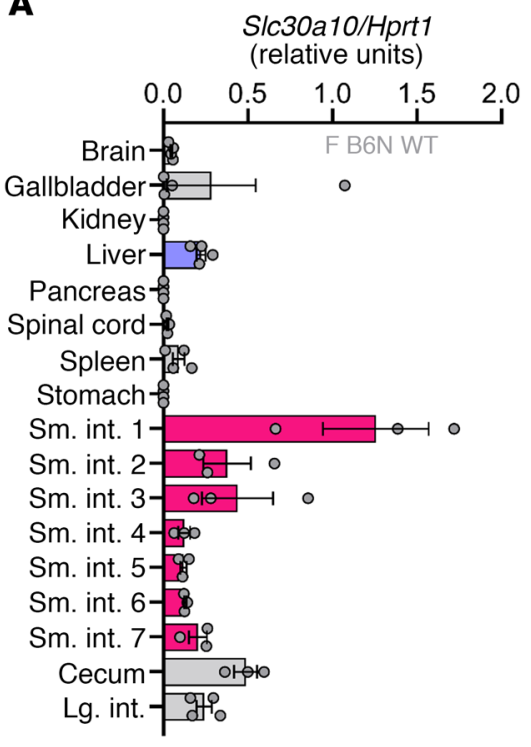

B

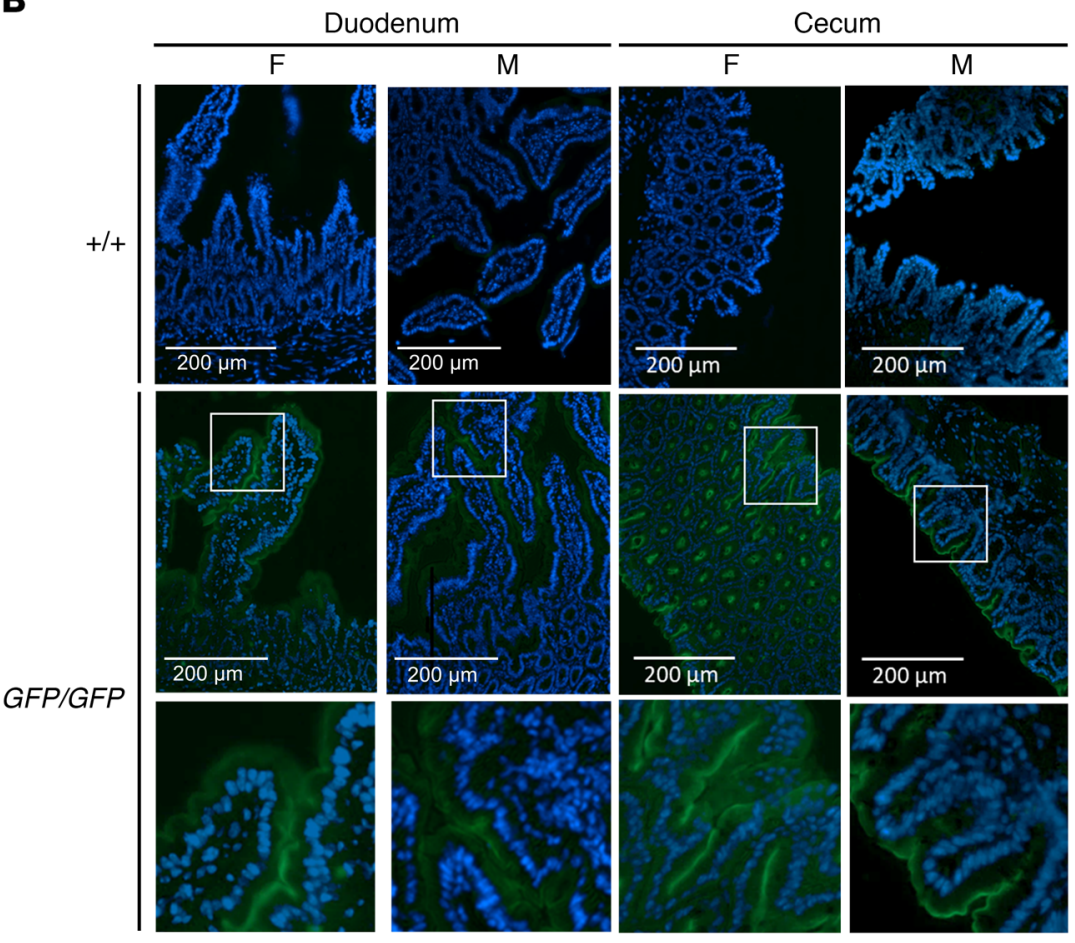

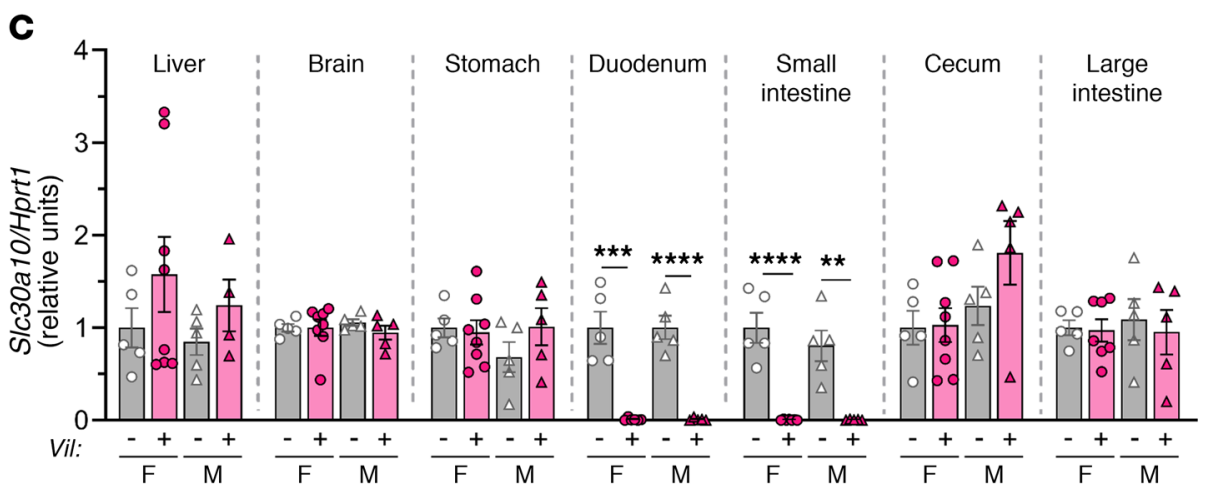

D

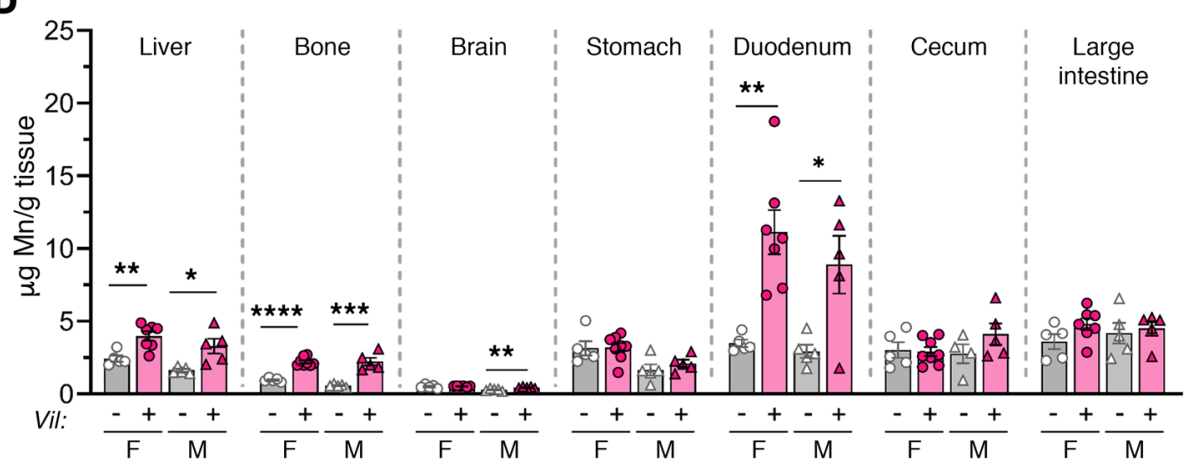

Figure 9. Enterocyte SIc30a10 contributes to the regulation of Mn levels. (A) Slc30a10/Hprt1 RNA ratios in 8-week-old female C57BL/6NJ mouse tissues. Small intestines were equally sectioned into 7 segments (sm. int. 1-7). (B) Fluorescence images of frozen duodenal and cecal sections from 2-month-old

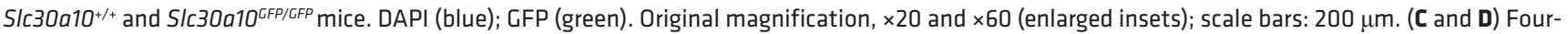
month-old SIc30a1010x/lox and SIc30a10/ox/lox Vil mice were analyzed for (C) tissue S/c30a10/Hprt1 RNA ratios, normalized to female lox/lox levels ("Duodenum" refers to the proximal $4 \mathrm{~cm}$ of small intestines; "small intestines" refers to the remaining organ) and (D) organ Mn levels. For all panels except B, data are presented as individual values and represent the mean \pm SEM. For $\mathbf{C}$ and $\mathbf{D}$, outliers (not shown) were identified by ROUT. Two-tailed $P$ values were calculated by unpaired $t$ test. Removal of 1 outlier in $\mathbf{C}$ and 2 outliers in $\mathbf{D}$ did not affect the designation of the comparisons with a $P$ value below 0.05 . Removal of the outlier in $\mathbf{D}$ (16.5 in female SIc30a10 ${ }^{10 x / 10 x}$ Vil liver) changed the $P$ value from $P>0.05$ to $P<0.01 . n=5-8$ replicates/group. ${ }^{*} P<0.05$, ${ }^{* *} P<$ $0.01,{ }^{* *} P<0.001$, and ${ }^{* * *} P<0.0001$. 


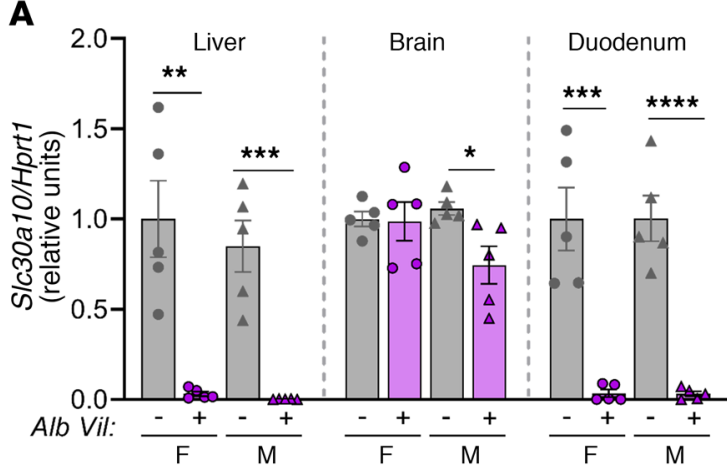

B
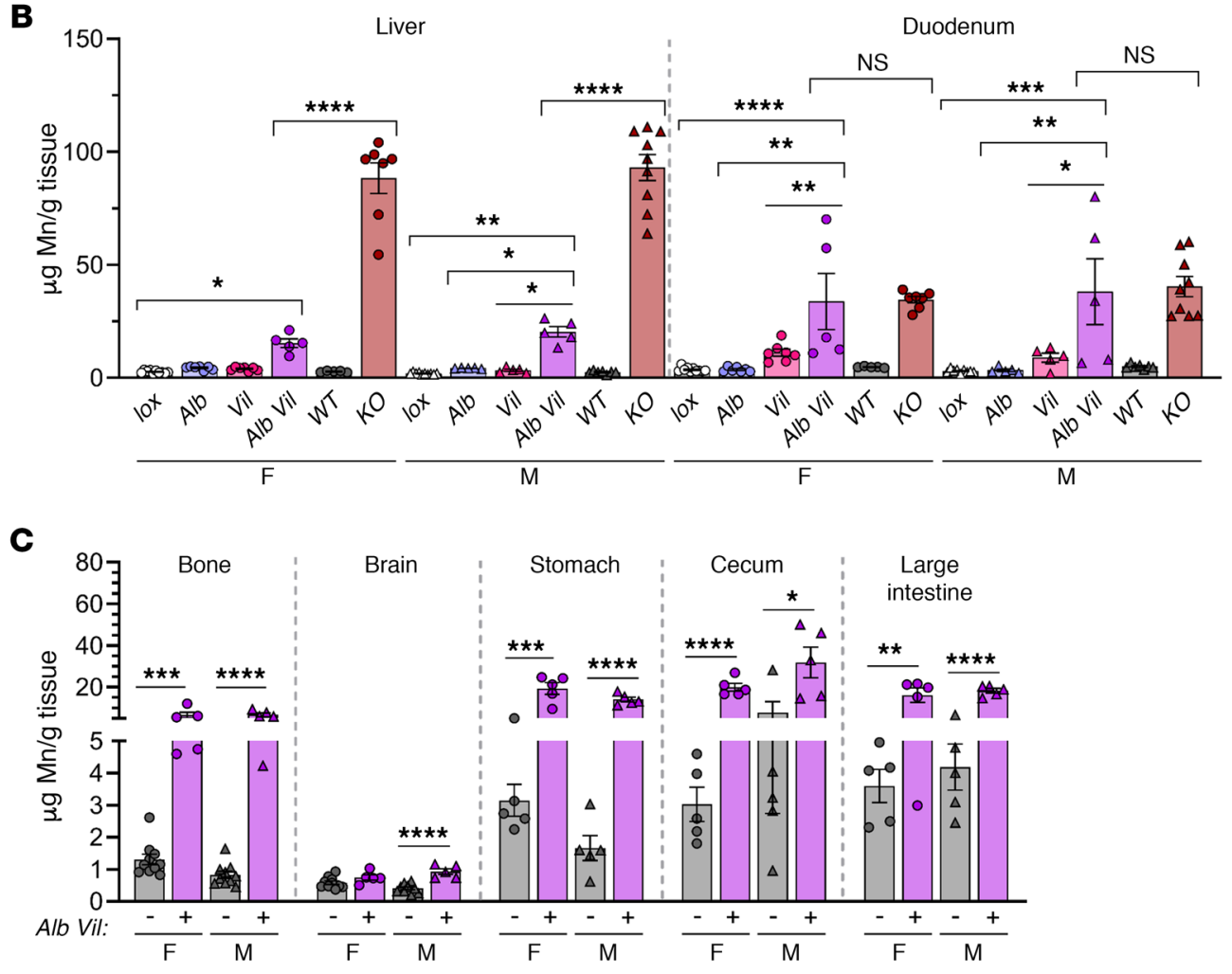

D

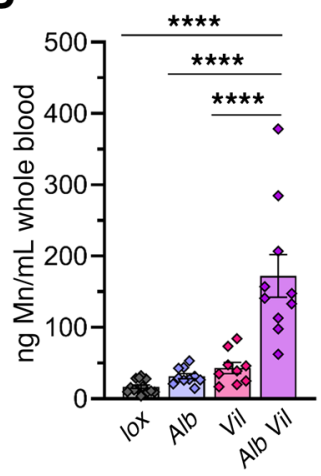

E

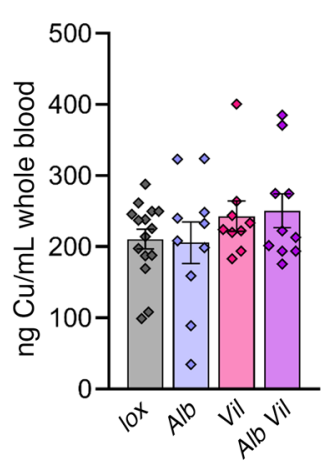

$\mathbf{F}$

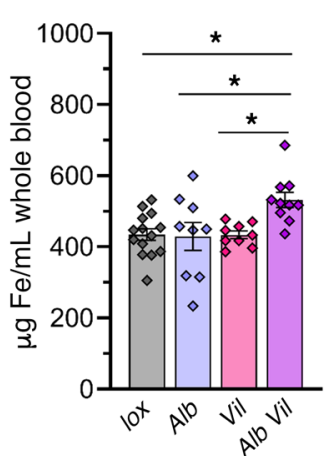

Figure 10. Hepatocyte and enterocyte SIc30a10 deficiency does not recapitulate the organ $\mathrm{Mn}$ excess seen in global SIc30a10 deficiency. SIc30a10 $10 x / 10 x$, Slc30a10 $0^{10 x / 10 x} \mathrm{Alb}$, SIc30a10 $10 x / 10 x$ Vil, and SIc30a10 $10 x / 10 x$ Alb Vil mice were analyzed at 4 months of age for (A) S/c30a10/Hprt1 RNA ratios, normalized to female lox/ lox levels, and (B and $\mathbf{C}$ ) organ $\mathrm{Mn}$ levels. Data from Figure 1D, Figure 7B, and Figure 9D were reproduced in B for reference. (D-G) Blood Mn (D), copper $(\mathbf{E})$, iron (F), and zinc (G) levels. Data for males and females were pooled. In all panels, data are presented as individual values and represent the mean \pm SEM. Outliers (data not shown) were identified by the ROUT method. In $\mathbf{A}$ and $\mathbf{C}$, 2-tailed $P$ values were calculated by unpaired $t$ test. In B, D-G, $P$ values were calculated by 1-way ANOVA with Tukey's multiple comparisons test. Only $P$ values involving Slc30a10 ${ }^{10 x / 10 x}$ Alb Vil mice are indicated in $\mathbf{B}$. No outliers were identified in A, B, or D-G. Removal of 1 outlier in $\mathbf{C}$ did not alter the designation of comparisons with a $P$ value below $0.05 . n=5-10$ replicates/group ${ }^{*} P<0.05,{ }^{* *} P<0.01,{ }^{* * *} P<0.001$ and ${ }^{* * *} P<0.0001$
$M n$ excretion by enterocytes in the small intestines is dependent on Slc30a10. To assess the function of Slc30a10 in enterocytes of the small intestines, we devised a surgical approach in which the hepatopancreatic duct (HPD) was ligated just proximal to its

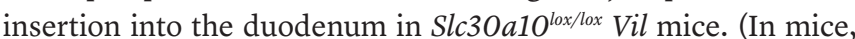
unlike in humans, the pancreatic duct merges with the common bile duct to form the HPD, which drains bile and pancreatic fluid into the small intestines.) Mice received an injection of ${ }^{54} \mathrm{Mn}$ via the portal vein, and then ${ }^{54} \mathrm{Mn}$ content in tissues and GI tract contents were assessed 1 hour later. We also performed this analysis in $\mathrm{Slc30a1O^{lox } / \text { lox }}$ Vil mice without ligation and in $\mathrm{Slc30a1O^{lox } / \text { lox }}$,

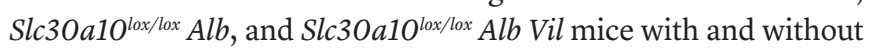


A

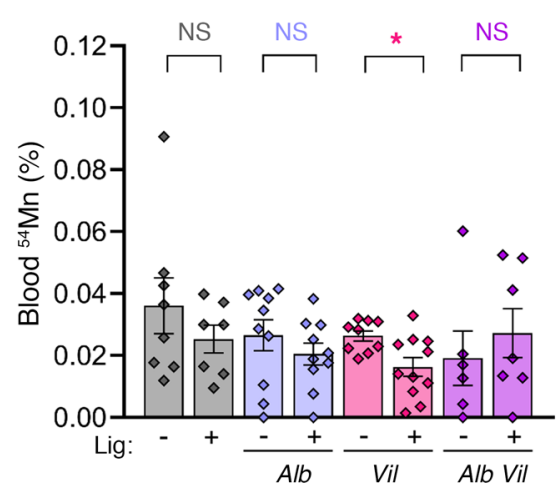

D

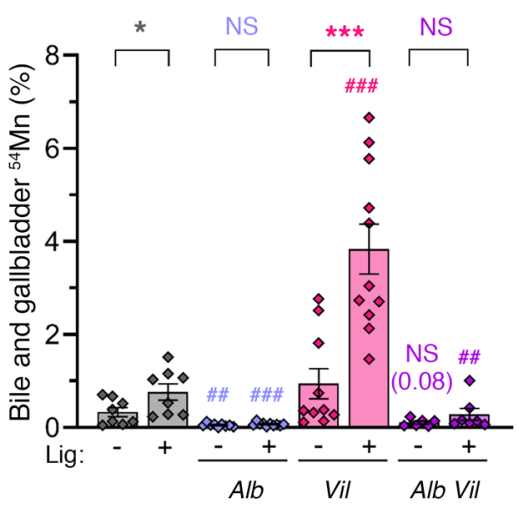

G

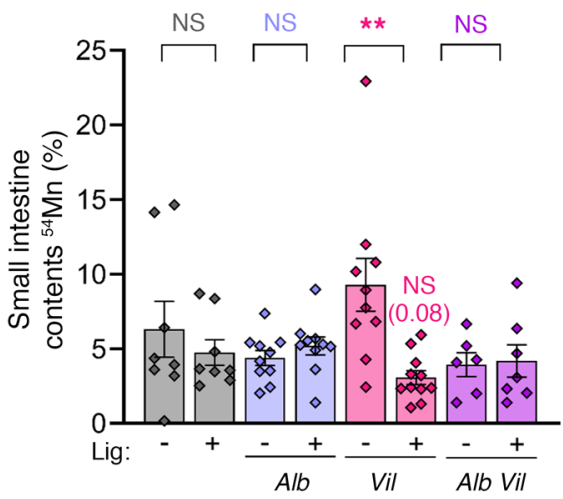

B

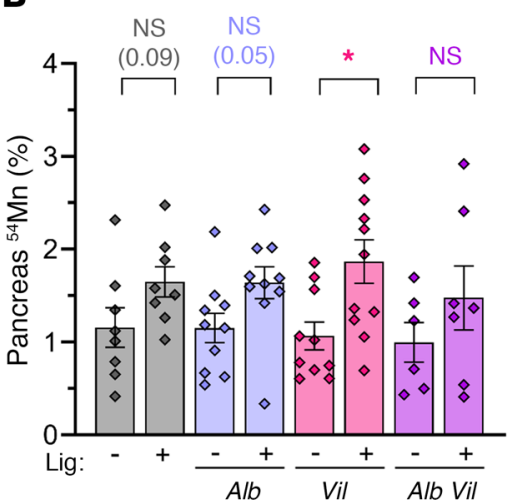

E

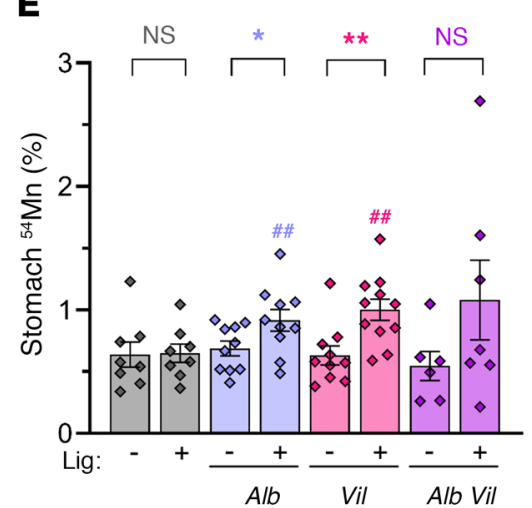

H

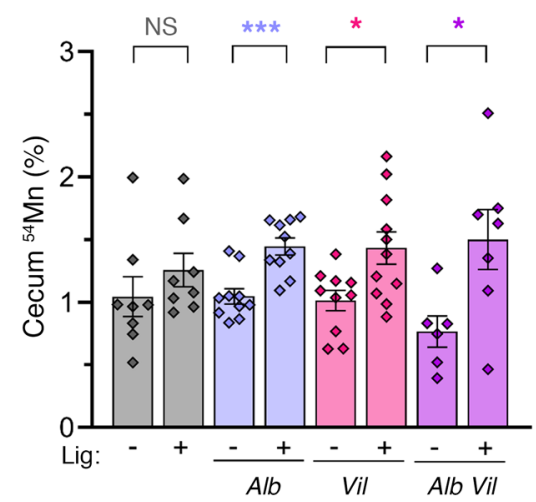

C

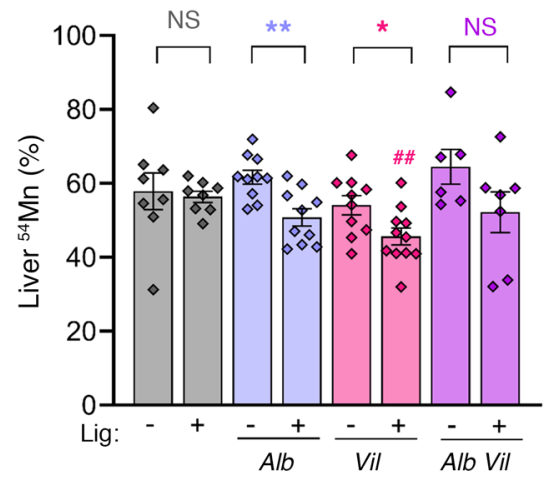

$\mathbf{F}$

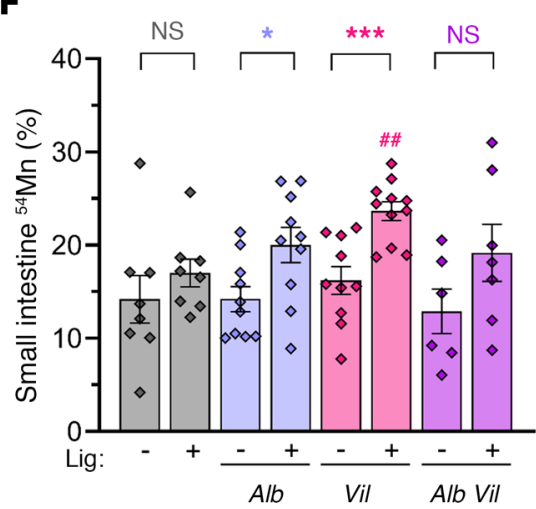

I

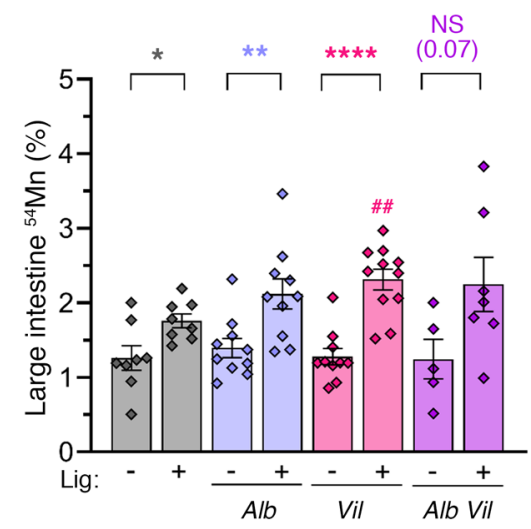

Figure 11. SIc30a10 in both hepatocytes and enterocytes contributes to the regulation of Mn levels. S/c30a10 $10 x / 10 x$ with or without $A / b$ and/or Vil underwent ${ }^{54} \mathrm{Mn}$ /fluorescein injection via the portal vein with (+) or without (-) prior HPD ligation (Lig). Samples were collected after 1 hour. Data for females and males were pooled. Data are presented as individual values and represent the mean \pm SEM. Outliers (not shown) were identified by ROUT. To compare groups with and without ligation or specific genotypes with lox/lox mice that underwent the same surgical treatment, 2-tailed $P$ values were calculated by unpaired $t$ test and are indicated above the brackets at the top of the panels: ${ }^{*} P<0.05,{ }^{* *} P<0.01,{ }^{* *} P<0.001$, and ${ }^{* * * *} P<0.0001$, for comparisons between mice of the same genotype with and without ligation; ${ }^{\#} P<0.01$ and ${ }^{\# \#} P<0.001$, for comparisons between the indicated mouse genotype and SIc30a10 $10 x / 10 x$ mice that underwent the same surgical treatment (ligation or no ligation). The values in parentheses represent $P$ values for select groups. Removal of the outlier in A (0.060 in female S/c30a10lox/lox Vil with ligation) changed the $P$ value from $P>0.05$ to $P<0.01$. No outliers were identified in B-I. $n=6-11$ replicates/group.

ligation. This approach was similar to that used to assess biliary Mn excretion except that the gallbladder was not cannulated and the bile duct was ligated at the hepatopancreatic ampulla, thereby blocking excretion from both the liver and pancreas. (Note that this approach would not occlude pancreatic accessory ducts that can be present in mice and drain fluid into small intestines without merging with the hepatobiliary tract; ref. 38.)
Blood ${ }^{54} \mathrm{Mn}$ levels were minimal in all groups, indicating that tissue ${ }^{54} \mathrm{Mn}$ levels largely reflected tissue ${ }^{54} \mathrm{Mn}$ uptake (Figure $11 \mathrm{~A}$ ). HPD ligation did not have a significant effect on pancreatic ${ }^{54} \mathrm{Mn}$ levels in most genotypes (Figure 11B) but did result in bile accumulation within the gallbladder (data not shown). Liver ${ }^{54} \mathrm{Mn}$ levels were

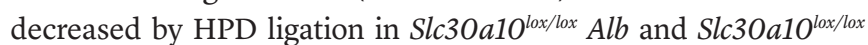
Vil mice (Figure 11C). ${ }^{54} \mathrm{Mn}$ levels, counted in gallbladder and bile 
contained within the gallbladder, were decreased in Slc30a10 lox/lox Alb mice and male Slc30a1O $0^{l o x} / l o x$ Alb Vil mice compared with Slc30a1O ${ }^{l o x} /$ lox mice, consistent with the essential role for Slc30a10 in biliary Mn export (Figure 11D). HPD ligation increased bile and

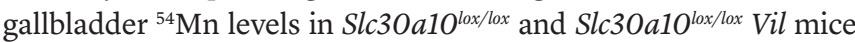
(Figure 11D). Although direct comparisons of ${ }^{54} \mathrm{Mn}$ in bile and gallbladder should be made carefully between groups with and without ligation due to the differences in bile volumes within gallbladders, the large increase in bile and gallbladder ${ }^{54} \mathrm{Mn}$ levels observed in Slc30a1O lox/lox Vil mice is likely too large to only reflect differences in bile volume and may reflect an essential role for Slc30a10, whereby ${ }^{54} \mathrm{Mn}$ can be excreted by enterocytes in the small intestines. This will be explored in more detail in the discussion.

We next assessed ${ }^{54} \mathrm{Mn}$ levels in specific organs within the GI tract. We found that stomach ${ }^{54} \mathrm{Mn}$ levels were increased in Slc30a1O ${ }^{l o x} /$ lox $\mathrm{Alb}$ and Slc30a1O $\mathrm{O}^{\text {lox } / \text { lox }}$ Vil mice compared with levels in Slc30a1O lox/lox mice with ligation and were increased by HPD

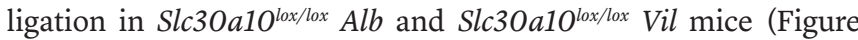
11E). ${ }^{54} \mathrm{Mn}$ levels in stomach contents were low or undetectable in all groups (data not shown). Ligation increased ${ }^{54} \mathrm{Mn}$ levels in the small intestines and decreased ${ }^{54} \mathrm{Mn}$ levels in small intestine contents in Slc30a10 ${ }^{\text {lox } / l o x}$ Vil mice (Figure 11, F and G). As ligation would block ${ }^{54} \mathrm{Mn}$ excretion from liver and pancreas into small intestines, this result is consistent with a role for enterocyte Slc30a10 in Mn excretion.

Given that Slc30a10 is expressed in the epithelial lining of the cecum (Figure 9B), we next assessed ${ }^{54} \mathrm{Mn}$ levels in both tissue and contents of cecum and large intestines. We observed increased, or a trend toward increased, ${ }^{54} \mathrm{Mn}$ levels in cecum and large intestines within most groups when comparing mice with and without ligation (Figure 11, $\mathrm{H}$ and I). ${ }^{54} \mathrm{Mn}$ levels in the contents of the cecum and large intestines varied largely within groups, which made interpretations difficult (data not shown). This variation may be attributed to the 1-hour time point, which may not be long enough to observe significant excretion of ${ }^{54} \mathrm{Mn}$ by these tissues.

\section{Discussion}

The goal of this study was to exploit Slc30a10-deficient mouse models to establish mechanisms of Mn homeostasis. Slc30 a10 mice recapitulated several disease phenotypes (Figures 1 and 2). Although mice in another model of Slc30a10 deficiency (31) developed aberrant thyroid function, a phenotype not reported in patients, our mice exhibited a milder thyroid phenotype, with normal serum thyroxine levels and a smaller average follicle size only in females (Figure 2, G-I). Differences in thyroid pathology between these models may be technical in origin. Whereas we deleted exon 3 and the coding region of exon 4 of Slc30a10, Hutchens et al. targeted exon 1. And whereas we used a pure C57BL/6N ES cell line, Hutchens et al. used a mixed C57BL/6 x $129 /$ Sv cell line. We harvested at 8 weeks of age, given that our mice did not routinely survive to 12 weeks of age; Hutchens et al. harvested at 6 weeks of age due to premature death of their mutant mice at 6 to 8 weeks of age. We used LabDiet 5010 (120 $\mu \mathrm{g}$ Mn/g chow); Hutchens et al. used PicoLab Rodent Diet 20 (84 $\mu \mathrm{g} \mathrm{Mn/g} \mathrm{chow).} \mathrm{Finally,} \mathrm{the} \mathrm{gut} \mathrm{microbiome,} \mathrm{which} \mathrm{can} \mathrm{vary}$ between animal facilities, may have affected phenotypes differently in our models (39).
In addition to the sex-specific difference in thyroid follicle size in $\mathrm{Slc3Oa1O}^{+/+}$versus Slc30 $\mathrm{O1O} \mathrm{KO}^{\mathrm{KO}}$ mice, we also observed sex-specific differences in biliary copper levels and bile flow rates (Figure 5, E and G). Although some of this may be attributed to intra-sex variability, other studies have reported sex-specific differences in Mn levels in individuals living near ferro-Mn industries, in rats fed diets deficient or adequate in $\mathrm{Mn}$, and in children with polymorphisms in Mn transporters (26, 40, 41). More research is required to understand the impact of sex on metal homeostasis in health and disease.

As mentioned above, the goal of this study was to establish mechanisms of Mn homeostasis. Two of our findings demonstrate that Slc30a10 is essential not only for Mn excretion but specifically for export of Mn by liver into bile. First, fecal ${ }^{54} \mathrm{Mn}$ and total biliary $\mathrm{Mn}$ levels were both inappropriately low in Slc3O a1O ${ }^{\mathrm{KO} / \mathrm{KO}}$ mice for their level of Mn excess (Figure 5, A and C) when compared with fecal ${ }^{54} \mathrm{Mn}$ and total biliary Mn levels in WT mice raised on a Mn-rich diet (Figure 4, A and G). Although biliary ${ }^{54} \mathrm{Mn}$ levels were minimal in $\mathrm{Slc30a1O^{KO } / \mathrm { KO }}$ mice after portal vein ${ }^{54} \mathrm{Mn}$ injection (Figure $5 \mathrm{H}$ ), ${ }^{54} \mathrm{Mn}$ may not have equilibrated with excess nonradioactive liver Mn during the 1-hour bile collection period. Second, in Slc30a10 ${ }^{l o x} /$ lox Alb mice, biliary levels of total $\mathrm{Mn}$ and ${ }^{54} \mathrm{Mn}$ after portal vein injection were minimal (Figure 8, $\mathrm{D}$ and F). Together, these data indicate that Slc30a10 exports Mn from hepatocytes into bile.

Our data also point to Slc30a10-independent mechanisms of $\mathrm{Mn}$ excretion. Although the inappropriately low fecal ${ }^{54} \mathrm{Mn}$ levels

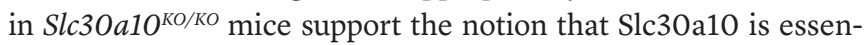
tial for Mn homeostasis, fecal ${ }^{54} \mathrm{Mn}$ levels were not decreased in Slc3Oa1O ${ }^{\mathrm{KO} / \mathrm{KO}}$ mice compared with fecal levels in $\mathrm{Slc} \mathrm{Oa} 1 \mathrm{O}^{+/+}$mice (Figure 5A). Whether these Slc30a10-independent mechanisms of fecal Mn excretion involve the Mn importer Slc39a14 or another metal transporter remains to be determined. Similarly, although

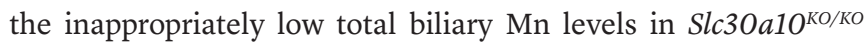
mice support the notion that Slc30a10 is essential for hepatobiliary $\mathrm{Mn}$ excretion, we found that total biliary Mn levels were similar between $\mathrm{Slc} \mathrm{Oa} \mathrm{OO}^{+/+}$and $\mathrm{Slc3Oa1O^{KO } / \mathrm { KO }}$ mice (Figure 5C). This indicates that some level of hepatobiliary Mn excretion can occur independently of Slc30a10, which may involve other metal transporters or paracellular Mn transport into bile $(42,43)$.

Although Slc30a10 lox/lox Alb mice had impaired biliary Mn excretion, they only developed minimal excess tissue Mn, similar to the findings of a previous study involving Slc30a10-deficient mice (16). One potential explanation is delayed gene inactivation (Supplemental Figure 6D), as previously reported for the Alb transgene (44). This implies that expression of liver Slc30a10 in developing mice plays a critical role in Mn homeostasis. Another potential explanation is compensation by Slc30a10 in another organ. The intestines, specifically the duodenum and jejunum, have been implicated in Mn excretion (45). Our studies demonstrate that Slc30a10 expression in both hepatocytes and enterocytes of the small intestines contribute to Mn homeostasis (Figures 9-11). Notably, Mn levels in small intestines were similar in

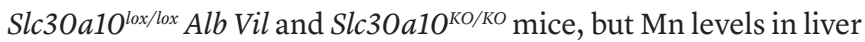

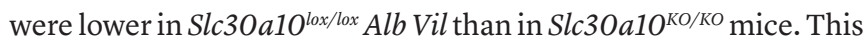
may reflect the idea that the small intestines have a much lower storage capacity for $\mathrm{Mn}$ than the liver. This also suggests that 
an additional stress is required to create severe liver Mn excess. This stress could include earlier onset of hepatocyte Slc30a10 deficiency or Slc30a10 deficiency in another organ such as the cecum and/or large intestines. (The Vil transgene only inactivated Slc30a1O in small intestines; Figure 9C.) This notion is supported by the observation that depletion of Slc30a10 in the digestive system using the endoderm-specific Foxa3-Cre transgene results in comparable Mn levels to a global Slc30a10-deficient mouse in all organs except brain (16). Since endoderm-specific Slc30a10deficient mice retain brain Slc30a10 expression, the lack of severe Mn excess in these mice suggests that brain Slc30a10 regulates Mn levels locally, not systemically (16). However, the specific role of Slc30a10 in the brain, i.e., Mn export from distinct cell types, remains to be established.

To evaluate Mn excretion in mice with enterocyte Slc30a10 deficiency, we used a surgical approach in which the HPD duct was ligated, the portal vein was injected with ${ }^{54} \mathrm{Mn}$, and tissues and contents of each GI tract region were collected 1 hour later and counted. This experiment produced several observations. First, when Slc30a10 was deficient in enterocytes, ${ }^{54} \mathrm{Mn}$ accumulated in the hepatobiliary tract, as evidenced by increased bile and gallbladder ${ }^{54} \mathrm{Mn}$ levels in ligated Slc30a10 ${ }^{\text {lox } / l o x}$ Vil mice (Figure 11D). The relatively mild increase in bile and gallbladder ${ }^{54} \mathrm{Mn}$ levels in $\mathrm{Slc30a1O^{lox } / l o x}$ mice with and without ligation suggests that when the HPD is ligated yet Slc30a10 is still expressed in other organs such as the small intestines, other routes for ${ }^{54} \mathrm{Mn}$ excretion can be rapidly implemented. Second, ligation in Slc3Oa1O ${ }^{\text {lox } / l o x}$ Vil mice led to decreased ${ }^{54} \mathrm{Mn}$ levels in the luminal contents of the small intestines (Figure 11G). This most likely reflects the notion that ${ }^{54} \mathrm{Mn}$ excretion into the lumen of the small intestines is impaired when the hepatobiliary system is acutely blocked and Slc30a10 is deficient in small intestine enterocytes. In contrast, ligation had no impact on ${ }^{54} \mathrm{Mn}$ levels in small intes-

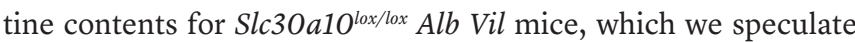
may reflect the ability of these mice, which were analyzed at 4 months of age, to adapt chronically to tissue-specific Slc30a10 deficiency by recruiting other routes of ${ }^{54} \mathrm{Mn}$ excretion such as the cecum and large intestines. Last, although ${ }^{54} \mathrm{Mn}$ levels in the luminal contents of the cecum and large intestines were undetectable or erratic (data not shown), we observed increased ${ }^{54} \mathrm{Mn}$ levels or a trend toward increased ${ }^{54} \mathrm{Mn}$ levels in the cecum and large intestines following ligation in almost all genotypes (Figure 11, $\mathrm{H}$ and I). Since Slc30a10 expression in these organs is retained in Slc30a1O lox/lox Vil mice, we propose that the cecum and large intestines may contribute to fecal Mn excretion. However, the 1-hour time period used in this experiment was insufficient to observe ${ }^{54} \mathrm{Mn}$ excretion by these organs.

Overall, this study demonstrates that Slc30a10 is essential for hepatobiliary Mn excretion and significantly contributes to intestinal Mn excretion. Since the discovery of SLC30A10 deficiency in 2012, mutations in 2 other Mn transporters have been identified in patients with Mn-related diseases. SLC39A14 is essential for Mn import into cells. Mutations in SLC39A14 lead to severe Mn excess (46). Slc39a14-deficient mice have increased Mn deposition in the brain leading to neurotoxic effects, with milder Mn accumulation in the liver, gallbladder, and GI tract, suggesting an impairment in systemic Mn elimination (18-20). Mutations in SLC39A8 have been reported in inherited Mn deficiency (47). Recent findings indicate that Slc39a8 is localized to the hepatocyte canalicular membrane and regulates systemic Mn homeostasis by reclaiming Mn from bile (21).

With consideration of these findings, our view of the molecular basis of Mn homeostasis is as follows: $\mathrm{Mn}$ is absorbed through the intestinal epithelium, however the essential factors have not been identified. The iron transporters SLC11A2 and SLC40A1 may contribute to this absorption process, however data supporting a critical role are lacking $(7-12,14)$. In intestinal enterocytes, SLC39A14 at the basolateral membrane and SLC30A10 at the apical membrane limit Mn absorption and/or enhance intestinal $\mathrm{Mn}$ excretion $(15,16)$. Once absorbed, $\mathrm{Mn}$ is transported to the liver for distribution to other tissues, storage, or use within liver, or excreted from the body. SLC39A14 is the primary Mn importer in the liver (18-20), whereas our data indicate that SLC30A10 is essential for Mn excretion into bile. Once in bile, Mn can be reimported into hepatocytes by SLC39A8 (21) or transported to the small intestines, where it can either be reabsorbed through enterohepatic circulation (45) or eliminated via feces. Although the mechanisms that govern Mn homeostasis are becoming more evident, the regulatory mechanisms that ensure Mn excretion by SLC39A14 and SLC30A10 in conditions of Mn excess and Mn retention by SLC39A8 in conditions of Mn deficiency have yet to be established. Presumably, regulatory mechanisms also exist to ensure that Mn is not excreted by SLC39A14 and SLC30A10 in conditions of Mn deficiency and that $\mathrm{Mn}$ is not retained by SLC39A8 in conditions of Mn excess. Additionally, since deficiency in all 3 of these transporters leads to severe disease, the importance of monitoring SNPs in these genes in populations sensitive to Mn toxicity may be critical. Such populations include miners and welders exposed to Mn-rich fumes or particulates, individuals who drink water with high Mn levels, or patients who receive $\mathrm{Mn}$-supplemented total parenteral nutrition.

\section{Methods}

Care and generation of mice. Mice were bred and maintained in the animal care facility at Brown University. Mice were group-housed in ventilated cage racks, maintained on a 12-hour light/12-dark cycle with controlled temperature and humidity, and provided standard chow (LabDiet 5010; 120 ppm Mn) and water ad libitum. Littermates of the same sex were randomly assigned to experimental groups.

The generation of Slc3Oa10 neo/neo mice was performed in the Mouse Transgenic and Gene Targeting Facility at Brown University. A targeting vector was purchased from the Knockout Mouse Project Repository at the University of California Davis. This vector carried FRT sites flanking $\beta$-gal and neomycin resistance genes inserted between Slc30a10 exons 2 and 3 and loxP sites flanking exon 3 and the coding region of exon 4. The vector was electroporated into C57BL6/NJM8. F6 ES cells. Appropriately targeted clones were screened by PCR and Southern blotting (data not shown) and microinjected into albino B6 (C57BL/6J-Tyrc-2J) blastocysts. Chimeric mice were bred with C57BL6/NJ mice (The Jackson Laboratory) to generate progeny carrying PCR-positive mutant alleles, indicative of germline transmission. Correct transgene insertion was confirmed by PCR using primer pairs flanking the FRT-loxP insertion upstream of exon 3 (5'-TCTCATGCTGGAGTTCTTCG-3, 5'-CAGCACTTGAGAGGCTGAGA-3') 
and flanking the loxP site downstream of the stop codon in exon 4 (5'-ACTGCCTCTGGCTCTCGTC-3'， 5'-TAGCATGCACAAGCCCTGTA-3'). Mice carrying the targeted allele $\left(\right.$ Slc30a10 $\left.{ }^{+/ n e o}\right)$ were bred with C57BL6/NJ mice expressing Flp recombinase under the direction of the $\beta$-actin promoter (The Jackson Laboratory) to generate mice with a conditional-ready $\left(\mathrm{Slc}_{3} \mathrm{Oa1O^{+/ } / \text { lox }}\right)$ allele in which $\beta$-gal and neomycin resistance genes were excised, leaving only loxP sites flanking exons 3 and the coding region of exon 4 .

To confirm appropriate Slc30a10 modifications, frozen liver samples from Slc3Oa1O neo/neo and Slc3Oa1O lox/lox mice underwent wholegenome sequencing (GENEWIZ). Genomic DNA was extracted and analyzed by NanoDrop 2000 and Qubit dsDNA Assay. DNA was processed using the Illumina TruSeq Paired-End Sequencing workflow, with whole-genome sequencing performed on an Illumina HiSeq in high-output mode with $2 \times 150$ bp paired-end configuration. Adapter sequences were trimmed and low-quality bases removed from sequence ends. Raw data, with approximately 400,000,000 reads, were analyzed using Geneious 10 (Biomatters). Sequences were paired and aligned against the predicted Slc30a10 sequence using the low-sensitivity setting with up to 5 iterations. (The predicted Slc30a10 sequence was constructed from the GRCm38 assembly of the Mus musculus strain C57BL/6J [Ensembl.org] and the targeting vector sequence from the Knockout Mouse Project repository.) A consensus sequence of the alignment was generated and aligned to the predicted Slc30a1O sequence, confirming Slc30a10 neo/neo sequence fidelity except for a 1-bp deletion upstream of the start codon, a 2-bp deletion and a 1-bp insertion upstream of the lacZ gene, and a 1-bp insertion upstream of the neomycin resistance gene. This also confirmed $S l c 30 a 10^{\operatorname{lox} / l o x}$ sequence fidelity except for the same 1-bp deletion upstream of the start codon observed in Slc30a10 nеo/neo. No sequence changes were noted in exons, exon-intron junctions, or loxP sequences. Raw sequence data will be provided upon request.

To generate mice with global Slc30a10 deficiency $\left(S_{c} 30 a 1 O^{K O / K O}\right)$,

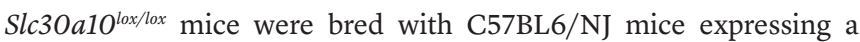
$\beta$-actin promoter-driven Cre recombinase (The Jackson Laboratory). Slc30a10 $0^{l o x / l o x}$ mice carrying the transgene were bred together to generate transgene-free $\mathrm{Slc3Oa} \mathrm{OO}^{\mathrm{KO} / \mathrm{KO}}$ mice. The presence of the $\mathrm{Slc3Oa1O^{lox }}$ allele was confirmed using a forward primer (5'-GTGTGTGAATGCATGGGTGT-3') complementary to the region of intron 2 upstream of the FRT site and a reverse primer (5'-GTAGCACTGCCAGTTACACG-3') located in exon 3 downstream of the FRT site. The Slc30a1O ${ }^{K O}$ allele, carrying a deletion of exon 3 and the coding region of exon 4, was confirmed using a forward primer (5'-GTGTGTGAATGCATGGGTGT-3') complementary to the region of intron 2 upstream of the FRT site and a reverse primer (5'-TAGCATGCACAAGCCCTGTA-3') complementary to exon 4 downstream of the loxP site.

To generate mice with hepatocyte-specific Slc30a10 deficiency

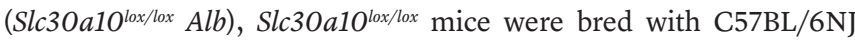
mice expressing an albumin promoter-driven Cre recombinase (Alb) (The Jackson Laboratory). To generate mice with hepatocyte-specific Slc40a1 and Slc30a10 deficiency (Slc40a1 fl/fl Slc30a10 ${ }^{\text {lox/lox }} \mathrm{Alb}$ ), 129S6/SvEvTac Slc4Oa $1^{f l / f l}$ mice (The Jackson Laboratory) were bred

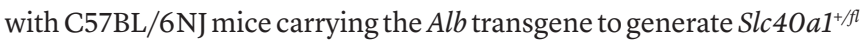

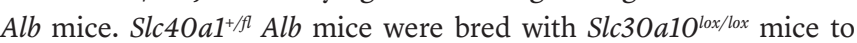
generate hepatocyte-specific Slc40a1- and Slc30a10-deficient mice

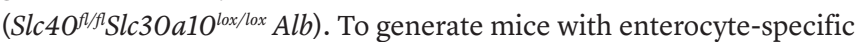
Slc30a10 deficiency (Slc30a1O ${ }^{\text {lox } / \text { lox }} \mathrm{Vil}$ ), Slc30a1O ${ }^{\text {lox } / \text { lox }}$ mice were bred with C57BL/6NJ mice expressing a villin promoter-driven Cre recombinase (Vil) (The Jackson Laboratory). The transgene genotype was determined through PCR assays at Transnetyx.

Mice expressing Slc30a10-GFP fusions (Slc30a10 ${ }^{\text {GFP/GFP }}$ ) were created by in-frame insertion of an mClover3 cDNA (AddGene) and a 3xFLAG epitope sequence immediately before the stop codon of Slc30a10. Gene editing was achieved by pronuclear injection of spCas 9 mRNA (TriLink), in vitro-transcribed sgRNA, and a donor plasmid into 1-cell C57BL/6N embryos $(48,49)$. Two sgRNAs (AAACAGTACTCATTTTTAGT and TCAGACATTTTGAATTGTGT) were designed (www.benchling.com) to introduce doublestranded breaks around the Slc30a1O stop codon. The donor plasmid for homology-directed repair was constructed by assembling a 0.8-kb left homology arm, a (GGGGS) linker, mClover3, another (GGGGS) ${ }_{2}$ linker, 3xFLAG, and a 1.5-kb right homology arm into pUC57-Simple (GenScript). Founder mice were identified by long-range PCR using primer pairs with 1 primer external to the homology arms (5'-LR-PCR: 5'-CGGATCTCCCTATGTTGTCC-3', 5'-GAACTTGTGGCCGTTTACG-3'; 3'-LR-PCR: 5'-ACAACGTTGAGGACGGCAG-3', 5'-CCTAGGCTCAAGCAACTCTTC-3'). Amplicons were sequenced to confirm targeting. Founder mice were bred with WT C57BL/6NJ mice for germline transmission of the targeted allele and generation of heterozygous progeny, which were bred together to generate $\mathrm{Slc} \mathrm{Oa} 1 \mathrm{O}^{+/+}$and $\mathrm{Slc} 3 \mathrm{Oa} 1 \mathrm{O}^{\mathrm{GFP} / \mathrm{GFP}}$ mice. The genotype was confirmed using primer pairs in exon 4 flanking the insertion site (5'-AAGGGAGCAGGGACAGACTC-3', 5'-TAGCATGCACAAGCCCTGTA-3') and complimentary to the mClover3 insert (5'-AAGGGAGCAGGGACAGACTC-3', 5'-GTCCTCCTTGAAGTCGATGC-3').

Adult C57BL/6NJ WT mice (The Jackson Laboratory) were bred in-house and provided LabDiet 5010 chow (120 ppm Mn) and water ad libitum. The progeny were weaned onto a control Mn (10 ppm Mn, 100 ppm iron) or high-Mn (2400 ppm Mn, 100 ppm iron) diet (Envigo). Littermates of the same sex were randomly assigned to experimental groups. Mice were provided the assigned diet and water ad libitum until tissue was harvested at 8 weeks of age.

Tissue and blood harvest. Tissue and blood were harvested

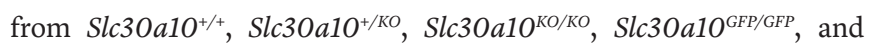
Slc3Oa1O $\mathrm{KO}_{\text {GFP }}$ mice at 8 weeks of age. Tissue and blood from

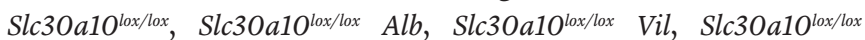

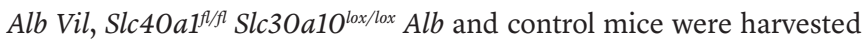
at 4 months of age. Mice were anesthetized by i.p. injection of ketamine-xylazine. Blood was collected by retro-orbital puncture using microhematocrit capillary tubes (Fisherbrand) and serum/blood collection tubes (BD Microtainer). Blood was first collected using heparinized capillary tubes into $\mathrm{K}_{2}$ EDTA-coated collection tubes for complete blood counts (Scil VetAbcPlus). Blood was next collected using nonheparinized capillary tubes into serum collection tubes; after 30 minutes at room temperature, the tubes were centrifuged at $10,000 \mathrm{~g}$ for 10 minutes and supernatants flash-frozen and stored at $-80^{\circ} \mathrm{C}$ until analysis. Additional blood was collected using heparinized capillary tubes into lithium-heparin tubes and stored at $-80^{\circ} \mathrm{C}$ until analysis. Mice were euthanized by cervical dislocation. Tissues were collected for histological and metal, DNA, RNA, and protein analysis. Tissues for histological analysis were fixed in 10\% PBS overnight and stored in $70 \%$ ethanol at $4^{\circ} \mathrm{C}$ until processing. (See below for details on fluorescence microscopy of Slc30a10 ${ }^{G F P / G F P}$ tissues.) All other tissues were flash-frozen and stored at $-80{ }^{\circ} \mathrm{C}$ until analysis. Serum thyrox- 
ine levels were measured using T4 ELISA (Life Technologies, Thermo Fisher Scientific).

$M R I$. Mice were fasted for 4 hours prior to imaging to clear GI tract contents. $\mathrm{T}_{1}$-weighted structural images were acquired using a Siemens 3 Tesla Prisma scanner. Mice were placed in a 16-channel wrist/ hand receive array. Two mice, side by side, were imaged at a time. Following scout image acquisition, a 3D $\mathrm{T}_{1}$-MPRAGE sequence was used for structural imaging. The field of view was $84 \mathrm{~mm}$, with an in-plane reconstruction matrix of $256 \times 256$ producing an in-plane resolution of $330 \mu \mathrm{m}$. Slice thickness was $330 \mu \mathrm{m}$ (104 slices), providing 3D isotropic resolution. The repetition time was $2700 \mathrm{~ms}$, with an inversion time of $1100 \mathrm{~ms}$. Echo time was $3.88 \mathrm{~ms}$, and the read flip angle was $9^{\circ}$. The receive bandwidth was $210 \mathrm{~Hz} /$ pixel. Parallel imaging was performed using GRAPPA with an acceleration factor of 2 . The scan time was 8 minutes 5 seconds on average. The final images were viewed using OsiriX Lite (Pixmeo).

RNA analysis. Tissue (100-200 mg) was homogenized in TRIzol (Invitrogen, Thermo Fisher Scientific) using $0.5 \mathrm{~mm}$ RNase-free zirconium beads and a Bullet Blender (Next Advance). Samples underwent chloroform extraction, isopropanol precipitation, a 70\% ethanol wash, and pellet resuspension in RNase-free water, as recommended by the TRIzol protocol. RNA concentrations and A260:A280 ratio were measured using NanoDrop (Thermo Fisher Scientific).

For most RNA analyses, the following protocol was used. Standards were made by serially diluting mixtures of control and experimental samples, and then processed identically as experimental samples. Samples underwent DNase treatment and cDNA synthesis using the High Capacity cDNA Reverse Transcription Kit with RNase Inhibitor (Life Technologies, Thermo Fisher Scientific). qPCR was performed using PowerUP SYBR Green Master Mix (Thermo Fisher Scientific). The following primer pairs were used: Hprt1 (5'-GCCCCAAAATGGTTAAGGTT-3'， 5'-TGGCAACATCAACAGGACTC-3', spanning exons 6-9); Slc30a10 (5'-AGAGACTGCTGTCATCTTGCTG-3'， 5'-TGTGCACTTCATGCACACTG-3'， spanning intron 3 between exons 3 and 4); Slc39a14 (5'-GGAACCCTCTACTCCAACGC-3', 5'-ATGGTTATGCCCGTGATGGT-3', spanning exons 5-7); Slc39a8 (5'-CAACGCAAAGCCCAGTCTTT-3', 5'-GCGTTTGAGAAAAGAGTCCCAA-3', spanning exons 3 and 4). Primers were designed using Primer3 and Geneious, with amplicon fidelity con-

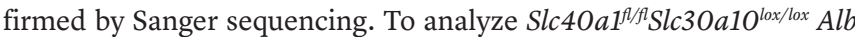
tissue RNA levels, TaqMan gene expression assays and Master Mix (Thermo Fisher Scientific) were used to measure Slc30a10, Slc40a1, and Hprt1 expression. To analyze RNA levels in parenchymal and nonparenchymal liver cell populations, TaqMan assays and Master Mix were used to measure expression of Slc30a10, Slc4Oa1, albumin (hepatocytes), Tek (sinusoidal endothelial cells), Pdgfrb (stellate cells), and Adgre1 (Kupffer cells).

Metal measurements. Tissue (10-500 mg) was digested in 500$1000 \mu \mathrm{L} 70 \%$ trace-metal-grade nitric acid (Termo Fisher Scientific) at $65^{\circ} \mathrm{C}$ for 2 hours and then diluted 25-fold in MilliQ water (MilliporeSigma) and analyzed by inductively coupled plasma atomic emission spectroscopy (ICP-AES) (Thermo Fisher Scientific, iCAP 7400 DUO) or graphite furnace atomic absorbance spectroscopy (GF-AAS) (PerkinElmer, AAnalyst 600). Blood and bile were digested with 2 and 4 volumes of nitric acid, respectively. Most samples were analyzed by ICP-AES; blood and bile were analyzed by GF-AAS. For ICP-AES, a series of standards were analyzed and sample values extrapolated from the generated curve. A quality control standard (Inorganic Ventures, IV-28) was run every 10 samples to assess changes in sensitivity. If the tissue size was small or metal levels low, GF-AAS was used. Standards were measured to create a standard curve. To correct for variations in sensitivity, a quality control standard was analyzed every 10 samples (NIST, SRM 1640a). Irrespective of instrumentation, a correction curve was calculated on the basis of quality control analysis and a correction factor applied to each sample to control for changes in instrument sensitivity.

Tissue microscopy. Slc3Oa10 ${ }^{+/+}$and Slc30a10 GFP/GFP tissues were fixed in $4 \%$ paraformaldehyde at $4^{\circ} \mathrm{C}$ for 1 hour, rinsed in PBS, transferred to $15 \%$ sucrose/PBS, and incubated at $4^{\circ} \mathrm{C}$ for 3 to 5 hours or until the tissues sank. Tissues were transferred to $30 \%$ sucrose/PBS and incubated at $4^{\circ} \mathrm{C}$ as before. Tissues were embedded in OCT (Thermo Fisher Scientific) on dry ice in tissue-embedding molds (Polysciences) and stored at $-80^{\circ} \mathrm{C}$ until sectioning. After trimming blocks at $30 \mu \mathrm{m}, 8-\mu \mathrm{m}$ sections were collected on Superfrost Plus slides (Fisherbrand) and stored at $-80^{\circ} \mathrm{C}$ until imaging. For imaging, slides were brought to room temperature and then washed twice in PBS for 10 minutes. DAPI $(0.5 \mathrm{~mL} 0.5 \mu \mathrm{g} / \mathrm{mL})$ (Invitrogen, Thermo Fisher Scientific) in PBS was placed on each slide, which was then incubated in the dark at room temperature for 5 minutes. Slides were rinsed twice in PBS for 10 minutes. Imaging was performed using the EVOS FL system (Thermo Fisher Scientific).

Immunofluorescence was performed on $8-\mu \mathrm{m}$ frozen sections. Sections were fixed in ice-cold methanol for 10 minutes followed by washing with PBS and blocking (10\% goat serum, 1\% BSA in PBS) for 1 hour at room temperature. Sections were incubated overnight with or without MDR1/ABCB1 antibody (Novus Biologicals, catalog NBP267667) in blocking buffer at $4^{\circ} \mathrm{C}$ without agitation. Slides were washed in PBS followed by incubation with a secondary antibody (goat antirabbit IgG, Alexa Fluor 594, Invitrogen, Thermo Fisher Scientific, catalog A-11012) in blocking buffer for 1 hour at room temperature. Slides were washed in PBS and mounted using VECTASHIELD mounting media containing DAPI (Vector Laboratories). All incubations were carried out in a humidified chamber with exposure to light minimized to avoid photobleaching.

To estimate the average thyroid follicle size, scanned images of H\&E-stained thyroid were analyzed using ImageJ (NIH). Results, including all technical replicates within biological replicates, were graphed using GraphPad Prism software.

Isolation of primary hepatocytes. Hepatocytes were isolated from 8-week-old C57BL/6J female mice (50). The portal vein was catheterized in an anesthetized mouse. The liver was perfused with $10 \mathrm{~mL}$ perfusion buffer (HBSS, $5 \mathrm{mM}$ HEPES, $0.5 \mathrm{mM}$ EDTA) and $10 \mathrm{~mL}$ collagenase solution (HBSS, $5 \mathrm{mM}$ HEPES, $0.5 \mathrm{mM} \mathrm{CaCl}, 0.5 \mathrm{mg} / \mathrm{mL}$ collagenase) at $2 \mathrm{~mL} / \mathrm{min}$. Immediately after the start of perfusion, the inferior vena cava was clipped; during perfusion with collagenase, the vena cava was clamped for 10 seconds every minute. The liver was removed, gently dispersed, and then strained through a 100- $\mu$ m nylon strainer. The strained cell suspension was centrifuged at $50 \mathrm{~g}$ for $3 \mathrm{~min}$ utes. The pellet was resuspended in $40 \%$ Percoll and centrifuged at $200 \mathrm{~g}$ for 5 minutes at $4^{\circ} \mathrm{C}$ to pellet viable hepatocytes. The supernatant was centrifuged at $500 \mathrm{~g}$ for 5 minutes at $4^{\circ} \mathrm{C}$, then the pellet was resuspended in PBS, $1 \mathrm{mM}$ EDTA, and 2\% FBS, mixed with an equal volume of $40 \%$ iodixanol, overlaid with PBS, 1 mM EDTA, and 2\% FBS, and then centrifuged at $1500 \mathrm{~g}$ for 25 minutes. Nonparenchymal cells were taken from the interface and pelleted at $500 \mathrm{~g}$ for 4 minutes at $4^{\circ} \mathrm{C}$. 
${ }^{54} \mathrm{Mn}$ excretion studies including radioactive and nonradioactive bile collection. To assess Mn excretion, $4 \mu \mathrm{Ci}^{5}{ }^{5} \mathrm{MnCl}_{2}$ (Perkin Elmer) was diluted in $100 \mu \mathrm{L}$ PBS and immediately retro-orbitally injected into mice anesthetized with isoflurane. Mice were placed in metabolic cages (Tecniplast) overnight for 16 hours for collection of urine and feces. (A pilot experiment in which urine and feces were collected for up to 16 hours after injection indicated that ${ }^{54} \mathrm{Mn}$ excretion peaked at 12 hours, with similar values at 16 hours; data not shown.) Mice were euthanized by cervical dislocation. ${ }^{54} \mathrm{Mn}$ levels were measured using the Triathler Gamma Counter with an external NaI well-type crystal detector (Hidex). Samples were counted in a $50-\mathrm{mL}$ conical tube for 15 seconds. Body ${ }^{54} \mathrm{Mn}$ levels were measured with mice positioned head-first in a $50-\mathrm{mL}$ conical tube. Tissues including liver, pancreas, spleen, kidney, heart, lung, muscle, bone, brain, stomach, small intestine, cecum, and large intestine were counted individually in a $1.5-\mathrm{mL}$ tube placed in a $50-\mathrm{mL}$ conical tube to normalize the distance from the detector. GI tract contents were removed prior to tissue counts. Remaining carcass was counted in a 50-mL conical tube. Urinary and fecal ${ }^{54} \mathrm{Mn}$ levels were measured in a $1.5-\mathrm{mL}$ tube. The percentage of excretion was calculated as the sum of urinary or fecal radioactivity as a percentage of the sum of all radioactivity.

For some studies, bile was collected after mice were injected with ${ }^{54} \mathrm{Mn}$ and housed in metabolic cages overnight using a modification of published methods (51-53). Mice were injected i.p. with $0.1 \mathrm{mg} / \mathrm{kg}$ buprenorphine and treated with ophthalmic ointment. Anesthesia was induced with $3 \%$ isoflurane using a vaporizer (VetEquip) and maintained with $1 \%-2 \%$ isoflurane, with the levels of anesthesia monitored by toe pinch throughout the procedure. Mice were injected s.c. with $0.5 \mathrm{~mL}$ normal saline and $0.5 \mathrm{~mL} 5 \%$ glucose, and then placed onto Deltaphase Isothermal Pads (Braintree Scientific). Abdominal fur was removed using commercial hair remover and skin disinfected with betadine and 70\% ethanol. A midline incision was made through the abdominal wall, followed by a transverse incision crossing the initial incision to permit full exposure of the abdominal cavity. The xiphoid process was clamped and the other end of the clamp fixed in modeler's clay to facilitate gallbladder visualization. The common bile duct was ligated using a 6-0 braided silk suture distal to the junction of hepatic and cystic ducts and proximal to the pancreas. Connective tissue attached to the gallbladder was cut to mobilize the gallbladder. A pre-tied 4-0 braided silk suture was placed around the base of the gallbladder. In the free end of the gallbladder, an incision was made through which $\mathrm{PE}-10$ polyethylene tubing (Instech Laboratories) was inserted. The pre-tied suture was tightened around the gallbladder to secure the tubing. Tegaderm was applied over the abdominal cavity to minimize dehydration. Normal saline was applied to the abdominal cavity intermittently throughout the procedure. Bile was collected for 1 hour, and then mice were euthanized by decapitation. Bile was transferred from the tubing into a microcentrifuge tube and centrifuged at $2000 \mathrm{~g}$ for 2 minutes to remove cells or debris and then transferred into a new $1.5-\mathrm{mL}$ centrifuge tube. ${ }^{54} \mathrm{Mn}$ levels were measured by gamma counting. Tissues including liver, pancreas, spleen, kidney, heart, lung, brain, stomach, small intestine, cecum, and large intestine were counted individually in $1.5-\mathrm{mL}$ tubes. GI tissues were counted with and without contents. Remaining carcass was counted in a $50-\mathrm{mL}$ conical tube. Urinary and fecal ${ }^{54} \mathrm{Mn}$ levels were measured in $1.5-\mathrm{mL}$ tubes. The percentage of excretion was calculated as the sum of urinary or fecal radioactivity as the percentage of the sum of all radioactivity.

Nonradioactive bile collection was performed as described above except that no ${ }^{54} \mathrm{Mn}$ was administered. Collected bile was stored at $-20^{\circ} \mathrm{C}$ until further analysis.

To assess the rate of ${ }^{54} \mathrm{Mn}$ biliary excretion, mice were prepared for bile collection as described above. Once bile flow into the gall-

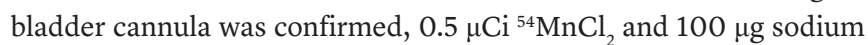
fluorescein (MilliporeSigma) in $10 \mu \mathrm{L}$ total volume were injected into the portal vein using a NANOFIL-10 syringe and a 35-gauge needle (World Precision Instruments). Fluorescein was included to confirm portal vein injection, common bile duct ligation, and gallbladder cannulation. Bile volume was noted every 5 minutes by observing the distance traveled by the bile fluid front in the gallbladder cannula. After 60 minutes of collection, the tubing was sectioned into 5-minute intervals, and ${ }^{54} \mathrm{Mn}$ levels in each section were measured. ${ }^{54} \mathrm{Mn}$ levels were measured in whole bodies and harvested tissues of euthanized mice after bile collection.

To determine whether Slc30a10 was required for Mn excretion by the small intestines, an approach similar to that described above to assess 54Mn biliary excretion was used except for 2 key differences. First, the bile duct was ligated at the site of the junction of the HPD with the small intestines, and second, the gallbladder was not cannulated.

Statistics. Statistical analysis was performed using GraphPad Prism 8 (GraphPad Software). Data are presented as individual values and represent the mean \pm SEM. $P$ values of less than 0.05 were considered statistically significant. To compare 2 groups, 2 -tailed $P$ values were calculated using an unpaired $t$ test. To compare more than 2 groups, $P$ values were calculated using 1-way ANOVA with Tukey's multiple comparisons test. To compare slopes of lines, linear regression was used. To compare thyroid follicle sizes, 2-tailed $P$ values were calculated by nested $t$ test. Outliers were identified using the GraphPad ROUT (robust regression and outlier removal) method $(Q=1 \%)$ and removed as stated in the figure legends.

Study approval. The studies were approved by the Institutional Animal Care and Use Committee of Brown University.

Contact for reagents and resource sharing. The authors are willing to distribute all materials, data sets, and protocols. The corresponding author holds responsibility for responding to requests and providing information regarding reagents and resource sharing. Further information and requests for resources and reagents should be directed to the corresponding author.

\section{Author contributions}

TBB was responsible for conceptualization of the study. CJM, MP, HLC, MED, CH, MAP, and TBB were responsible for the study methodology. CJM, MP, HLC, MED, CH, MAP, LCR, MAS, DBR, and $\mathrm{TBB}$ performed experiments. $\mathrm{CJM}$ and $\mathrm{TBB}$ wrote the original draft of the manuscript. TBB reviewed and edited the manuscript. CJM and TBB performed imaging and analysis. TBB supervised the study. CJM and TBB were responsible for funding acquisition. Evaluation of histology was performed by DBR. This manuscript reflects the views of DBR on work conducted as an approved Outside Activity and should not be construed as representing the official views or policies of the US FDA, nor should mention of trade names or commercial products constitute US FDA endorsement or recommendation for use. 


\section{Acknowledgments}

Work was supported by NIH grants DK84122 (to TBB), DK110049 (to TBB), DK117524 (to CJM), ESO07272-24 T32 (to CJM), and GM077995 T32 (to HLC). Creation of Slc30a10-deficient mice in the Mouse Transgenic and Gene Targeting Facility at Brown University was supported by NIH grant P30 GM103410. We acknowledge Christoph Schorl and the Genomics Core for assistance with
qPCR; Michael Worden and the MRI facility at Brown University for assistance with imaging, and Joseph Orchardo and David Murray for assistance with metal analysis.

Address correspondence to: Thomas Bartnikas, Brown University, 70 Ship Street, Box GE5, Providence, Rhode Island 02912, USA. Phone: 401.863.3478; Email: thomas_bartnikas@brown.edu.
1. Lucchini RG, Martin CJ, Doney BC. From manganism to manganese-induced parkinsonism: a conceptual model based on the evolution of exposure. Neuromolecular Med.2009;11(4):311-321.

2. Racette BA. Manganism in the 21st century: the Hanninen lecture. Neurotoxicology. 2014;45:201-207.

3. Aschner JL, Aschner M. Nutritional aspects of manganese homeostasis. Mol Aspects Med. 2005;26(4-5):353-362.

4. Burkhard PR, Delavelle J, Du Pasquier R, Spahr L. Chronic parkinsonism associated with cirrhosis: a distinct subset of acquired hepatocerebral degeneration. Arch Neurol. 2003;60(4):521-528.

5. Santos D, Batoreu C, Mateus L, Marreilha Dos Santos AP, Aschner M. Manganese in human parenteral nutrition: considerations for toxicity and biomonitoring. Neurotoxicology. 2014;43:36-45.

6. Roth JA. Homeostatic and toxic mechanisms regulating manganese uptake, retention, and elimination. Biol Res. 2006;39(1):45-57.

7. Shawki A, et al. Intestinal DMT1 is critical for iron absorption in the mouse but is not required for the absorption of copper or manganese. Am J Physiol Gastrointest Liver Physiol. 2015;309(8):G635-G647.

8. Seo YA, Wessling-Resnick M. Ferroportin deficiency impairs manganese metabolism in flatiron mice. FASEB J. 2015;29(7):2726-2733.

9. Chen $\mathrm{P}$, et al. Manganese homeostasis in the nervous system. J Neurochem. 2015;134(4):601-610.

10. Mitchell CJ, Shawki A, Ganz T, Nemeth E, Mackenzie B. Functional properties of human ferroportin, a cellular iron exporter reactive also with cobalt and zinc. Am J Physiol, Cell Physiol. 2014;306(5):C450-C459.

11. Choi EK, Nguyen TT, Iwase S, Seo YA. Ferroportin disease mutations influence manganese accumulation and cytotoxicity. FASEB $J$. 2019;33(2):2228-2240.

12. Jin L, et al. Mice overexpressing hepcidin suggest ferroportin does not play a major role in Mn homeostasis. Metallomics. 2019;11(5):959-967.

13. Yin $Z$, et al. Ferroportin is a manganeseresponsive protein that decreases manganese cytotoxicity and accumulation. J Neurochem. 2010;112(5):1190-1198.

14. Madejczyk MS, Ballatori N. The iron transporter ferroportin can also function as a manganese exporter. Biochim Biophys Acta. 2012;1818(3):651-657.

15. Scheiber IF, Wu Y, Morgan SE, Zhao N. The intestinal metal transporter ZIP14 maintains systemic manganese homeostasis. J Biol Chem. 2019;294(23):9147-9160.

16. Taylor CA, et al. SLC30A10 transporter in the digestive system regulates brain manganese under basal conditions while brain SLC30A10 protects against neurotoxicity. J Biol Chem. 2019;294(6):1860-1876.

17. Chen P, Parmalee N, Aschner M. Genetic factors and manganese-induced neurotoxicity. Front Genet. 2014;5:265.

18. Jenkitkasemwong S, et al. SLC39A14 deficiency alters manganese homeostasis and excretion resulting in brain manganese accumulation and motor deficits in mice. Proc Natl Acad Sci U S A. 2018;115(8):E1769-E1778.

19. Aydemir TB, et al. Metal transporter Zip14 (Slc39a14) deletion in mice increases manganese deposition and produces neurotoxic signatures and diminished motor activity. JNeurosci. 2017;37(25):5996-6006.

20. Xin Y, et al. Manganese transporter Slc39a14 deficiency revealed its key role in maintaining manganese homeostasis in mice. Cell Discov. 2017;3:17025.

21. Lin W, et al. Hepatic metal ion transporter ZIP8 regulates manganese homeostasis and manganese-dependent enzyme activity. J Clin Invest . 2017;127(6):2407-2417.

22. Quadri M, et al. Mutations in SLC30A10 cause parkinsonism and dystonia with hypermanganesemia, polycythemia, and chronic liver disease. Am J Hum Genet. 2012;90(3):467-477.

23. Stamelou M, et al. Dystonia with brain manganese accumulation resulting from SLC30A10 mutations: a new treatable disorder. Mov Disord. 2012;27(10):1317-1322.

24. Tuschl K, et al. Syndrome of hepatic cirrhosis, dystonia, polycythemia, and hypermanganesemia caused by mutations in SLC30A10, a manganese transporter in man. Am J Hum Genet. 2012;90(3):457-466.

25. Leyva-Illades D, et al. SLC30A10 is a cell surface-localized manganese efflux transporter, and parkinsonism-causing mutations block its intracellular trafficking and efflux activity. J Neurosci. 2014;34(42):14079-14095.

26. Wahlberg K, et al. Polymorphisms in manganese transporters show developmental stage and sex specific associations with manganese concentrations in primary teeth. Neurotoxicology. 2018;64:103-109.

27. Wahlberg K, et al. Common polymorphisms in the solute carrier SLC30A10 are associated with blood manganese and neurological function. Toxicol Sci. 2016;149(2):473-483.

28. $\mathrm{Ng} \mathrm{E}$, et al. Genome-wide association study of toxic metals and trace elements reveals novel associations. Hum Mol Genet. 2015;24(16):4739-4745.

29. Kim G, Lee HS, Seok Bang J, Kim B, Ko D, Yang M. A current review for biological monitoring of manganese with exposure, susceptibility, and response biomarkers. JEnviron Sci Health C Environ Carcinog Ecotoxicol Rev. 2015;33(2):229-254.
30. Burgdorf KS, et al. Association studies of novel obesity-related gene variants with quantitative metabolic phenotypes in a population-based sample of 6,039 Danish individuals. Diabetologia. 2012;55(1):105-113.

31. Hutchens S, et al. Deficiency in the manganese efflux transporter SLC30A10 induces severe hypothyroidism in mice. J Biol Chem. 2017;292(23):9760-9773.

32. Sato I, Matsusaka N, Kobayashi H, Nishimura Y. Effects of dietary manganese contents on $54 \mathrm{Mn}$ metabolism in mice. J Radiat Res. 1996;37(2):125-132.

33. Harada $\mathrm{M}$, et al. Biliary copper excretion in acutely and chronically copper-loaded rats. Hepatology. 1993;17(1):111-117.

34. Mercadante CJ, et al. The effect of high dose oral manganese exposure on copper, iron and zinc levels in rats. Biometals. 2016;29(3):417-422.

35. Liu C, et al. Hypothyroidism induced by loss of the manganese efflux transporter SLC30A10 may be explained by reduced thyroxine production. J Biol Chem. 2017;292(40):16605-16615.

36. Wang CY, Babitt JL. Liver iron sensing and body iron homeostasis. Blood. 2019;133(1):18-29.

37. Donovan A, et al. The iron exporter ferroportin/ Slc40a1 is essential for iron homeostasis. Cell Metab. 2005;1(3):191-200.

38. Dolenšek J, Rupnik MS, Stožer A. Structural similarities and differences between the human and the mouse pancreas. Islets. 2015;7(1):e1024405.

39. Parker KD, Albeke SE, Gigley JP, Goldstein AM, Ward NL. Microbiome composition in both wild-type and disease model mice is heavily influenced by mouse facility. Front Microbiol. 2018;9:1598.

40. Bauer JA, et al. Manganese in teeth and neurobehavior: sex-specific windows of susceptibility. Environ Int. 2017;108:299-308.

41. Conde Martel A, González Reimers E, Santolaria Fernández F, Castro Alemán V, Marchena Gómez J, Martínez Riera A. [Liver changes in protein malnutrition. An experimental study in rats]. Nutr Hosp. 1993;8(6):358-363.

42. Boyer JL. Mechanisms of bile secretion and hepatic transport. In: Andreoli TE, Hoffman JF, Fanestil DD, Schultz SG, eds. Membrane Transport Processes in Organized Systems. Boston, MA: Springer US; 1987:225-252.

43. Boyer JL. Bile formation and secretion. Compr Physiol. 2013;3(3):1035-1078.

44. Postic C, Magnuson MA. DNA excision in liver by an albumin-Cre transgene occurs progressively with age. Genesis. 2000;26(2):149-150.

45. Bertinchamps AJ, Miller ST, Cotzias GC. Interdependence of routes excreting manganese. $A m J$ Physiol. 1966;211(1):217-224.

46. Tuschl K, et al. Mutations in SLC39A14 disrupt 
manganese homeostasis and cause childhood-onset parkinsonism-dystonia. Nat Commun. 2016;7:11601.

47. Park JH, et al. SLC39A8 deficiency: a disorder of manganese transport and glycosylation. Am J Hum Genet. 2015;97(6):894-903.

48. Wang $\mathrm{H}$, et al. One-step generation of mice carrying mutations in multiple genes by CRISPR/ Cas-mediated genome engineering. Cell.
2013;153(4):910-918.

49. Yang H, Wang H, Shivalila CS, Cheng AW, Shi L, Jaenisch R. One-step generation of mice carrying reporter and conditional alleles by CRISPR/ Cas-mediated genome engineering. Cell. 2013;154(6):1370-1379.

50. Mohar I, Brempelis KJ, Murray SA, Ebrahimkhani $\mathrm{MR}$, Crispe IN. Isolation of non-parenchymal cells from the mouse liver. Methods Mol Biol.
2015;1325:3-17.

51. Pedreira F, Tepperman J. Bile flow rate and cholesterol conten in mice fed a gallstone-inducing diet. Am J Physiol. 1964;206:635-640.

52. Plaa GL, Becker BA. Demonstration of bile stasis in the mouse by a direct and an indirect method. JAppl Physiol. 1965;20(3):534-537.

53. Rubin DH, Eaton MA, Costello T. Reovirus type 1 is secreted into the bile. JVirol.1986;60(2):726-728. 